\title{
Distribution sensitivity in stochastic programming
}

\author{
Werner Römisch and Rüdiger Schultz \\ Sektion Mathematik, Humboldt-Universität Berlin, O-1086 Berlin, Germany \\ Received 31 December 1987 \\ Revised manuscript received 20 June 1989
}

In this paper, stochastic programming problems are viewed as parametric programs with respect to the probability distributions of the random coefficients. General results on quantitative stability in parametric optimization are used to study distribution sensitivity of stochastic programs. For recourse and chance constrained models quantitative continuity results for optimal values and optimal solution sets are proved (with respect to suitable metrics on the space of probability distributions). The results are useful to study the effect of approximations and of incomplete information in stochastic programming.

AMS 1980 Subject Classifications: 90C15, 90C31.

Key words: Stochastic programming, quantitative stability, recourse problem, chance constrained problem, probability metric.

\section{Introduction}

In the present paper we study the behaviour of stochastic programming problems with respect to (small) perturbations of the underlying probability distributions. Emphasis is placed on quantitative stability results for optimal values and sets of optimal solutions to stochastic programs. To explain our aim, let us consider the following rather general stochastic programming model

$$
\min \left\{\int_{Z} f(z, x) \mu(\mathrm{d} z): x \in \mathbb{R}^{m}, \mu(\{z \in Z: x \in X(z)\}) \geqslant p_{0}\right\}
$$

where $Z \subset \mathbb{R}^{s}$ is a Borel set, $f$ is a function from $Z \times \mathbb{R}^{m}$ to $\mathbb{R}, X$ is a set-valued mapping from $Z$ into $\mathbb{R}^{m}, p_{0} \in[0,1]$ is a prescribed probability level and $\mu$ is a probability distribution on $Z$. Note that stochastic programs with (linear and quadratic) recourse (cf. (3.3) and (3.4)) and programs with probabilistic (or chance) constraints (cf. (5.1)) fit into (1.1).

Motivated by a number of applications, it seems particularly desirable to establish the stability of stochastic programs with respect to perturbations of the underlying distribution $\mu$ in the sense of the topology of weak convergence on $\mathscr{P}(Z)-$ the

This research was presented in parts at the 4th International Conference on Stochastic Programming held in Prague in September 1986. 
space of all Borel probability measures defined on $Z$ (cf., e.g., [6]). Since we are aiming at quantitative stability results, we are interested in suitable metrics on $\mathscr{P}(Z)$ which (at least locally) metrize the topology of weak convergence (cf., e.g., $[16 ; 27$, Chapter 2]). As we will see, such metrics are the bounded Lipschitz metric $\beta$ for recourse models and the variational distance $\alpha_{\mathscr{B}}$ for models with chance constraints. These metrics are defined as follows (cf. $[16,27])$ :

$$
\begin{aligned}
& \beta(\mu, \nu):=\sup \left\{\left|\int_{Z} g(z) \mu(\mathrm{d} z)-\int_{Z} g(z) \nu(\mathrm{d} z)\right|: g: Z \rightarrow \mathbb{R},\|g\|_{\mathrm{BL}} \leqslant 1\right\}, \\
& \alpha_{\mathscr{B}}(\mu, \nu):=\sup \{|\mu(B)-\nu(B)|: B \in \mathscr{B}\}(\mu, \nu \in \mathscr{P}(Z)),
\end{aligned}
$$

where

$$
\|g\|_{\mathrm{BL}}:=\sup _{z \in Z}|g(z)|+\sup _{\substack{z, \tilde{z} \in Z \\ z \neq z}} \frac{|g(z)-g(\tilde{z})|}{d(z, \tilde{z})}
$$

$\mathscr{B}$ is a subset of the Borel $\sigma$-field $\mathscr{B}(Z)$ of $Z,(Z, d)$ is a separable metric space (with metric $d$ ).

It is known that $\beta$ metrizes the topology of weak convergence on $\mathscr{P}(Z)$ [16] and that a sequence $\left(\mu_{n}\right)$ in $\mathscr{P}(Z)$ converges to $\mu \in \mathscr{P}(Z)$ in the sense of the (pseudo-) metric $\alpha_{\mathscr{B}}$ if $\left(\mu_{n}\right)$ converges weakly to $\mu$ and if $\mathscr{B}$ is a $\mu$-uniformity class ([7] and Remark 5.2). For interrelations of $\beta$ and $\alpha_{\mathscr{B}}$ to other probability metrics on $\mathscr{P}(Z)$ we refer to the literature $[16,27]$ and to Remark 5.10. In this context we remark that, especially for the stability considerations in the recourse case, we will identify a subset of $\mathscr{P}(Z)$ for which the quantitative stability results (with respect to the metric $\beta$ ) can be expected (cf. Section 3).

Our approach to quantitative continuity of the (locally) optimal values and optimal solution sets to stochastic programming models relies on recent developments in the quantitative stability analysis of parametric optimization problems with parameters varying in metric spaces. In particular, we make use of results obtained by Klatte [35, 36].

On the one hand, our general results are applicable to the stability analysis of stochastic programs with incomplete information on $\mu$ (see Corollary 3.4, Remark 5.11). For investigations along this line we refer e.g. to $[17-20,23,51-54,56]$.

On the other hand, approximations to stochastic programs which arise when the (known) probability distribution $\mu$ is approximated by "simpler" (e.g. discrete) ones fit into our setting for sensitivity analysis. From the number of papers dealing with such approximations here we only quote $[8,24,28,31,32,37-39,50,57]$.

The papers by Kall [30], Robinson and Wets [46], Kaňková [34] and Römisch and Wakolbinger [49] concentrate on topics that have a lot in common with the questions addressed in the present paper.

Using the results of [45] the authors of [30] and [46] obtain similar results concerning qualitative stability of optimal values and optimal solution sets of fairly general recourse models in the presence of perturbations of the distribution $\mu$ in 
the sense of the topology of weak convergence on suitable subsets of $\mathscr{P}(Z)$. As distinct from this, in Sections 3 and 4 we investigate more specialized recourse models for which we obtain quantitative stability results under slightly sharper assumptions on the distributions.

In [30], questions concerning the stability of chance constrained models are addressed. As one of the challenging problems in this respect, the author mentions the lack of comprehensible and (easily) verifiable assumptions to assure stability of the model with respect to the topology of weak convergence on $\mathscr{P}(Z)$. In Section 5 , we take up this question and show that the desired stability can be expected at least for distributions $\mu$ which are "smooth" in a certain sense (Proposition 5.1 and Remark 5.2).

The results in [49] on quantitative stability of optimal values for linear recourse and chance constrained models are extended by the following investigations in a twofold manner: We obtain (quantitative) stability results for optimal solution sets and we consider more general problem classes.

The papers of Dupačová [17-20], Wang [53] and Garstka [23] are aiming at similar goals. The authors establish the sensitivity of optimal values and optimal solutions of stochastic programs with respect to changes in the (finite-dimensional) parameters of the underlying probability distribution. Dupačová and Wang employ versions of the implicit function theorem and obtain, under certain assumptions, results about the existence of (differentiable and Lipschitzian, respectively) solution trajectories for stochastic programs (as functions of the finite-dimensional parameters).

Our paper is organized as follows. In Section 2 we quote the quantitative stability results from parametric optimization (Theorem 2.5 and 2.6) which are essential for the remainder of the paper and which are due to Klatte. In Section 3 we apply a result on the continuity with respect to the metric $\beta$ for special functionals which are given by integrals (Theorem 3.1) to derive Hölder continuity of the optimal value function and upper semicontinuity of the optimal set mapping for general classes of recourse problems (Theorem 3.3). Under more restrictive assumptions we obtain in Section 4 quantitative continuity results for optimal solutions of recourse models with special structure that permits separability of the recourse function (Theorem 4.4 and 4.8). In Section 5 we derive stability results for general chance constrained models. In the main result of this section (Theorem 5.4) we give conditions under which the optimal values (optimal solution sets) of such models behave locally Lipschitzian (upper semicontinuous) with respect to a metric $\alpha_{\mathscr{B}}$ (with suitably chosen $\mathscr{B}$ ).

\section{On quantitative stability in parametric programming}

The classical approach to sensitivity of optimal values and optimal solutions for optimization problems depending on parameters relies on the implicit function 
theorem and its generalizations (see e.g. [21] and the references therein). The following example is to illustrate the necessity of an alternative approach when aiming at sensitivity results for stochastic programs whose parameters are the underlying distributions.

Example 2.1. Consider

$$
F(x, \mu)=\int_{Z} f(z, x) \mu(\mathrm{d} z)
$$

with

$$
f(z, x)=\min _{v^{+}, v^{-}}\left\{v^{+}+v^{-}: v^{+}-v^{-}=z-x, v^{+} \geqslant 0, v^{-} \geqslant 0\right\} .
$$

For a fixed distribution $\mu$, the minimization with respect to $x$ of $F$ over $\mathbb{R}^{1}$ is a stochastic linear programming problem with simple recourse. Let us take $Z \subset \mathbb{R}^{1}$ as the closed interval $\left[-\frac{1}{2}, \frac{1}{2}\right]$ and assume that all distributions $\mu$ under consideration have a continuous density $\theta_{\mu}$ on $Z$. Then one confirms that $\nabla_{x x} F(0, \mu)$, the second derivative (with respect to $x$ ) of $F(\cdot, \mu)$ at $x=0$, is equal to $2 \theta_{\mu}(0)$ (cf. the proof of Proposition 4.1). Now let $\mu_{0}$ be the uniform distribution on $Z$. Consider the sequence $\mu_{n}$ of distributions on $Z$ given by the densities

$$
\theta_{n}(t)= \begin{cases}\left(1-t_{n}\right)^{-1} & \text { if } t_{n} \leqslant|t| \leqslant \frac{1}{2}, \\ \left(t_{n}-t_{n}^{2}\right)^{-1}|t| & \text { if } 0 \leqslant|t| \leqslant t_{n},\end{cases}
$$

where $t_{n} \in\left(0, \frac{1}{2}\right)$ and $t_{n} \rightarrow 0$.

The sequence $\mu_{n}$ then weakly converges to $\mu_{0}$, since the densities are converging pointwise with the exception of a set with Lebesgue measure zero [6, Scheffe's Theorem].

We have that $\nabla_{x x} F\left(0, \mu_{0}\right)=2$ and $\nabla_{x x} F\left(0, \mu_{n}\right)=0$ for each $n$. The point $x_{0}=0$ is the (global) minimizer of $F\left(\cdot, \mu_{0}\right)$ over $\mathbb{R}^{1}$. Hence the function $\nabla_{x x} F(\cdot, \cdot)$ is not continuous at $\left(x_{0}, \mu_{0}\right)$. This lack of continuity prevents the application of the implicit function theorem as well as the exploitation of stability results for optimization problems with parameters varying in a metric space that have been obtained by Cornet and Vial [12] (assumption (v) at p. 1125 in [12] does not hold).

The next example shows that in our approach unique solvability is lost when perturbing the problem.

Example 2.2. Consider the function $F(x, \mu)$ as in Example 2.1. Let $\mu_{0}$ again be the uniform distribution on $Z=\left[-\frac{1}{2}, \frac{1}{2}\right]$ and let $\mu_{n}$ be the discrete distribution having mass $1 /(2 n)$ at each of the mass points $\pm(2 K+1) /(4 n)(K=0, \ldots, n-1), n \in \mathbb{N}$. One observes that the distribution functions of $\mu_{n}$ converges pointwise to the distribution function of $\mu_{0}$ as $n \rightarrow \infty$. Hence, the $\mu_{n}$ converge weakly to $\mu_{0}$ [6]. However, the minimizing set of $F\left(\cdot, \mu_{n}\right)$ is the closed interval $[-1 /(4 n), 1 /(4 n)]$. 
The material we are going to present next has been taken from the papers $[35,36]$ by Klatte. We refer the reader to those papers for proofs. We remark that the central notions introduced below already appear (sometimes slightly modified) in papers by Aubin [2] and Robinson [45].

Consider the optimization problem

$$
P(t) \quad \min _{x}\{f(x, t): x \in M(t)\}
$$

depending on a parameter $t$ varying in some nonempty metric space $(T, d)$.

Assumptions on the function $f: \mathbb{R}^{m} \times T \rightarrow \mathbb{R}$ and the multifunction $M$ from $T$ into $\mathbb{R}^{m}$ are specified below. Given $V \subset \mathbb{R}^{m}$ we set for $t \in T$ :

$M_{V}(t)=M(t) \cap \mathrm{cl} V$ ("cl" means closure),

$\varphi_{V}(t)=\inf \left\{f(x, t): x \in M_{V}(t)\right\}$,

$\psi_{V}(t)=\left\{x \in M_{V}(t): f(x, t)=\varphi_{V}(t)\right\}$.

By $\psi_{\mathrm{loc}}(t)$ we denote the set of all local minimizers of $P(t)$.

Definition 2.3. Given $P\left(t^{0}\right)$ for fixed $t=t^{0}$, a nonempty set $X \subset \mathbb{R}^{m}$ is called a complete local minimizing set (CLM set) for $f\left(\cdot, t^{0}\right)$ on $M\left(t^{0}\right)$ with respect to $V$, if $V$ is an open set of $\mathbb{R}^{m}$ such that $V \supset X$ and $X=\psi_{V}\left(t^{0}\right)$.

Our notation follows Robinson [45], who introduced the above concept for an extended real valued $f$. In Klatte's papers, $X$ is called a "strict local minimizing set" (SLM set). Later on we will briefly say that $\psi_{V}\left(t^{0}\right)$ is a CLM set for $f\left(\cdot, t^{0}\right)$ on $M\left(t^{0}\right)$ meaning that the set in question is a CLM set for $f\left(\cdot, t^{0}\right)$ on $M\left(t^{0}\right)$ with respect to $V$.

Examples for CLM sets are the set of global minimizers to $P\left(t^{0}\right)$ or strict local minimizing points to $P\left(t^{0}\right)$ (i.e. points $z \in \mathbb{R}^{m}$ satisfying: there exists an open neighbourhood $V$ of $z$ such that $f\left(z, t^{0}\right)<f\left(x, t^{0}\right)$ for all $\left.x \in\left(M\left(t^{0}\right) \cap \mathrm{cl} V\right) \backslash\{z\}\right)$. Note that a CLM set is always a subset of $\psi_{100}$.

Definition 2.4. A multifunction $M$ from $T$ into $\mathbb{R}^{m}$ is said to be pseudo-Lipschitzian* at $\left(x^{0}, t^{0}\right)$, where $t^{0} \in T$ and $x^{0} \in M\left(t^{0}\right)$, if there exist a neighbourhood $V$ of $x^{0}$ and two positive reals $\delta_{M}$ and $L_{M}$ such that

$$
(M(t) \cap V) \subset M\left(t^{0}\right)+L_{M} d\left(t, t^{0}\right) B_{m}
$$

and

$$
\left(M\left(t^{0}\right) \cap V\right) \subset M(t)+L_{M} d\left(t, t^{0}\right) B_{m}
$$

for all $t \in T$ such that $d\left(t, t^{0}\right)<\delta_{M} .\left(B_{m}\right.$ denotes the closed unit ball in $\mathbb{R}^{m}$.)

The above is a relaxation of the concept of a pseudo-Lipschitzian multifunction introduced by Aubin [2] (cf. also [47] and Section 5). 
Theorem 2.5. Consider the parametric program $P(t)$, fix some $t^{0} \in T$.

(i) Assume that there exists a bounded open subset $V$ of $\mathbb{R}^{m}$ and a nonempty subset $X$ of $V$ such that $X=\psi_{V}\left(t^{0}\right)$ (i.e. $X$ is a bounded CLM set for $f\left(\cdot, t^{0}\right)$ on $M\left(t^{0}\right)$ ).

(ii) Let the multifunction $M$ be closed-valued and closed at $t^{0}$.

(iii) Suppose $M$ is pseudo-Lipschitzian* at each pair $\left(x^{0}, t^{0}\right)$ belonging to $\psi_{v}\left(t^{0}\right) \times\left\{t^{0}\right\}$.

(iv) Suppose there are real numbers $p \in(0,1], L_{f}>0$ and $\delta_{f}>0$ such that $f(\cdot, t)$ is continuous and

$$
\left|f\left(x, t^{0}\right)-f(y, t)\right| \leqslant L_{f}\left(\|x-y\|+d\left(t, t^{0}\right)^{p}\right)
$$

for each $x, y \in \mathrm{cl} V$ and each $t \in T$ satisfying $d\left(t, t^{0}\right)<\delta_{f}$.

Then we have:

(a) The multifunction $\psi_{V}$ is upper semicontinuous (u.s.c.) at $t^{0}$, i.e. for each $\varepsilon>0$ there exists $\eta>0$ such that

$$
\psi_{V}(t) \subset \psi_{V}\left(t^{0}\right)+\varepsilon B_{m} \quad \text { whenever } d\left(t, t^{0}\right)<\eta .
$$

(b) There exist positive reals $\delta_{\varphi}$ and $L_{\varphi}$ such that $\psi_{V}(t)$ is a CLM set for $f(\cdot, t)$ on $M(t)$ and $\left|\varphi_{V}(t)-\varphi_{V}\left(t^{0}\right)\right| \leqslant L_{\varphi} d\left(t, t^{0}\right)^{p}$ whenever $d\left(t, t^{0}\right)<\delta_{\varphi}$.

The starting point for the following version of a quantitative continuity result for the mapping $\psi_{V}$ lies in the work done by Alt [1] and Auslender [3] for the standard nonlinear programming problem with differentiable and locally Lipschitzian data, respectively.

Theorem 2.6. Consider the parametric program $P(t)$, fix some $t^{0} \in T$.

(i) Assume that there exists a strict local minimizer $z \in \mathbb{R}^{m}$ of order $q \geqslant 1$ to $P\left(t^{0}\right)$, i.e. there exist real numbers $\rho>0$ and $\Delta>0$ such that

$$
f\left(x, t^{0}\right)>f\left(z, t^{0}\right)+\Delta\|x-z\|^{q}
$$

for all $x \in\left(M\left(t^{0}\right) \cap B(z, \rho)\right) \backslash\{z\}$, where $B(z, \rho)$ denotes the closed ball around $z$ with radius $\rho$.

(ii) Let the multifunction $M$ be closed-valued.

(iii) Suppose $M$ is pseudo-Lipschitzian* at $\left(z, t^{0}\right)$.

(iv) Suppose there are real numbers $p \in(0,1], L_{f}>0$ and $\delta_{f}>0$ such that $f(\cdot, t)$ is continuous and

$$
\left|f\left(x, t^{0}\right)-f(y, t)\right| \leqslant L_{f}\left(\|x-y\|+d\left(t, t^{0}\right)^{p}\right)
$$

for each $x, y \in B(z, \rho)$ and each $t \in T$ satisfying $d\left(t, t^{0}\right)<\delta_{f}$.

Then there exist positive reals $\varepsilon, L_{\psi}$ and $\delta_{\psi}$ such that with $V:=B(z, \varepsilon)$ one has for each $t \in T$ with $d\left(t, t^{\circ}\right)<\delta_{\psi}$ and for each $x \in \psi_{v}(t)$,

$$
\|z-x\|^{q} \leqslant L_{\psi} d\left(t, t^{0}\right)^{p} .
$$


Remark 2.7. The constants $L_{\varphi}$ and $L_{\psi}$ in the above theorems can be set to $C\left(1+L_{f}\right)$ for some constant $C>0$ (not depending on $L_{f}$ ).

The above theorems differ from previous stability results in the more general hypotheses on the continuity of $f$ and $M$. From the viewpoint of stochastic programming these generalizations are essential. As we will see later on, recourse functionals and multifunctions arising from chance constraints possess such continuity properties.

3. Quantitative continuity of expectation functionals and applications to stability of recourse problems

Let us start with a result that forms the basis for the analysis of the present section.

Let $(Z, d)$ be a separable metric space and $\mathscr{P}(Z)$ be the set of all Borel probability measures on $Z$ equipped with the bounded Lipschitz metric $\beta$ which metrizes the topology of weak convergence (cf. Section 1).

Let $h$ be a mapping from $Z$ into $\mathbb{R}$ such that

$$
|h(z)-h(\tilde{z})| \leqslant L(\max \{d(z, 0), d(\tilde{z}, 0)\}) \cdot d(z, \tilde{z})
$$

where $L: \mathbb{R}^{+} \rightarrow \mathbb{R}^{+}$is continuous and monotonically increasing, and $0 \in Z$ is some distinguished element.

For $p \in[1,+\infty)$ and $\mu \in \mathscr{P}(Z)$ let us define

$$
M_{p}(\mu):=\left[\int_{Z}(L(d(z, 0)) \cdot d(z, 0))^{p} \mu(\mathrm{d} z)\right]^{1 / p} .
$$

Theorem 3.1 [49, Theorem 2.1]. There exists $C>0$ such that for all $p \in(1,+\infty)$ and $\mu, \nu \in \mathscr{P}(Z)$ we have

$$
\begin{aligned}
& \left|\int_{Z} h(z) \mu(\mathrm{d} z)-\int_{Z} h(z) \nu(\mathrm{d} z)\right| \\
& \quad \leqslant C\left(1+M_{p}(\mu)+M_{p}(\nu)\right) \cdot \beta(\mu, \nu)^{1-1 / p} .
\end{aligned}
$$

This theorem asserts Hölder-continuity of the mapping (acting from $(\mathscr{P}(Z), \beta)$ into $\mathbb{R}$ )

$$
\mu \mapsto \int_{Z} h(z) \mu(\mathrm{d} z)
$$

with respect to all subsets of $\mathscr{P}(Z)$ that have the shape

$$
\left\{\mu \in \mathscr{P}(Z): M_{p}(\mu) \leqslant K\right\}
$$

with some a priori given constants $p \in(1,+\infty)$ and $K \in(0,+\infty)$. In what follows we exclusively consider the important special case that $Z$ is closed and belongs to some Euclidean space (with norm $\|\cdot\|)$ and $L$ admits a representation $L(t)=L_{0} \cdot t\left(L_{0}>0\right.$, 
$t \in \mathbb{R}^{+}$). In this situation Theorem 3.1 yields Hölder-continuity of the mapping (3.2) with respect to all subsets

$$
\mathscr{P}(Z ; p, K):=\left\{\mu \in \mathscr{P}(Z): \int_{Z}\|z\|^{2 p} \mu(\mathrm{d} z) \leqslant K\right\}
$$

of $\mathscr{P}(Z)$.

In their paper on stability of recourse-type problems, Robinson and Wets [46] impose the essential hypothesis that the (family of) recourse-integrands is uniformly integrable with respect to a family of probability measures, i.e. if $\mathscr{F}$ is a family of continuous real valued functions on $Z \subset \mathbb{R}^{s}$ and $\mathscr{P}$ is a family of Borel probability measures on $Z$ then $\mathscr{F}$ is called uniformly integrable with respect to $\mathscr{P}$ if for each $\varepsilon>0$ there is a compact set $A \subset Z$ such that for each $f \in \mathscr{F}$ and each $P \in \mathscr{P}$,

$$
\int_{Z \backslash A}|f(\xi)| P(\mathrm{~d} \xi)<\varepsilon .
$$

An estimate using Hölder's inequality now yields the following: If $\mathscr{F}$ is uniformly bounded and equi-Lipschitzian (w.r.t. the Lipschitz property (3.1)) and $\mathscr{P}$ is tight [6] and contained in some set $\mathscr{P}(Z ; p, K)$ then $\mathscr{F}$ is uniformly integrable in the above sense. The recourse integrands which will be considered in the present paper turn out to be uniformly bounded and equi-Lipschitzian on compact sets. Hence, such a family of integrands is uniformly integrable with respect to a set in $\mathscr{P}(Z ; p, K)$ formed by the members of a weakly converging sequence and its limit point. Thus, for the recourse problems considered below, our integrability assumption is more restrictive than the one used in [46]. This is not surprising considering the emphasis that we put on quantitative aspects.

We remark that similar relations may be established between our integrability assumption and those imposed by Kall in his paper [30]. Although these assumptions are more restrictive than other integrability assumptions encountered in stochastic programming, it, nevertheless, is still possible to fulfill the conditions induced by sets of the type $\mathscr{P}(Z ; p, K)$ in important applications:

Let $\mu \in \mathscr{P}\left(\mathbb{R}^{s} ; p, K\right)$ and $\tilde{\mu}$ be a discrete approximation of $\mu$ via conditional expectations (for details see $[8,31]$ ). Jensen's inequality then implies that also $\tilde{\mu} \in \mathscr{P}\left(\mathbb{R}^{s} ; p, K\right)$. Or let $\mu, \mu_{n} \in \mathscr{P}\left(\mathbb{R}^{s}\right)(n \in \mathbb{N})$ be Gaussian measures such that the $\mu_{n}$ converge weakly to $\mu$. Then for any $p \geqslant 1$ there exists $K=K(p, \mu)>0$ such that $\mu, \mu_{n} \in \mathscr{P}\left(\mathbb{R}^{s} ; p, K\right)$ for all $n \in \mathbb{N}$ (cf. [10]).

The exponent $1-1 / p$ arising on the right-hand side of the estimate in Theorem 3.1 is the best possible (cf. [49]). If in Theorem 3.1 the integrand $h$ is bounded on $Z$ or if the supports of the measures $\mu$ and $\nu$ belong to some bounded set then the estimate holds with exponent 1 on the right-hand side.

Next, we introduce stochastic programs with linear and quadratic recourse, respectively, which we study in the present section. Consider

$$
\min _{x}\left\{c^{\mathrm{T}} x+\int_{Z_{1}} f_{1}(z, x) \mu(\mathrm{d} z): x \in C\right\}
$$


where $C \subset \mathbb{R}^{m}$ is a nonempty convex polyhedron and $c \in \mathbb{R}^{m}$,

$$
\begin{aligned}
& f_{1}(z, x):=\min _{v}\left\{q^{\mathrm{T}} v: W v=a-T x, v \geqslant 0\right\}, \\
& z:=(q, a, T), \\
& Z_{1}=\left\{(q, a, T): q \in \mathbb{R}^{l}, a \in \mathbb{R}^{r}, T \in L\left(\mathbb{R}^{m}, \mathbb{R}^{r}\right),\left\{u \in \mathbb{R}^{r}: W^{\mathrm{T}} u \leqslant q\right\} \neq \emptyset\right\},
\end{aligned}
$$

$W \in L\left(\mathbb{R}^{l}, \mathbb{R}^{r}\right)$ such that for all $y \in \mathbb{R}^{r},\left\{v \in \mathbb{R}^{l}: W v=y, v \geqslant 0\right\}$ is nonempty, $\mu \in \mathscr{P}\left(Z_{1}\right)$.

In the literature the above problem is called a stochastic linear program with complete fixed (linear) recourse. According to the assumptions on $Z_{1}$ and $W$ the function $f_{1}$ is well defined and real-valued on $Z_{1} \times \mathbb{R}^{m}$. For details we refer to $[28,55]$. Furthermore, we are going to study linear stochastic programs with quadratic recourse which are given as follows:

$$
\min _{x}\left\{c^{\mathrm{T}} x+\int_{Z_{2}} f_{2}(z, x) \mu(\mathrm{d} z): x \in C\right\}
$$

where $C \subset \mathbb{R}^{m}$ is a nonempty convex polyhedron and $c \in \mathbb{R}^{m}$,

$$
\begin{aligned}
& f_{2}(z, x):=\max _{v}\left\{-\frac{1}{2} v^{\mathrm{T}} H v+(a-T x)^{\mathrm{T}} v: A v \leqslant b, v \geqslant 0\right\}, \\
& z:=(b, a, T), \\
& Z_{2}=\left\{(b, a, T): b \in \mathbb{R}^{r}, a \in \mathbb{R}^{l}, T \in L\left(\mathbb{R}^{m}, \mathbb{R}^{l}\right),\left\{v \in \mathbb{R}^{l}: A v \leqslant b, v \geqslant 0\right\} \neq \emptyset\right\},
\end{aligned}
$$

$H \in L\left(\mathbb{R}^{l}, \mathbb{R}^{l}\right)$ symmetric and positive definite, $\mu \in \mathscr{P}\left(Z_{2}\right)$.

The assumptions on $Z_{2}$ and $H$ guarantee $f_{2}$ to be a well defined real-valued function on $Z_{2} \times \mathbb{R}^{m}$.

For more details on stochastic programs with quadratic recourse we refer to the paper [48] which has inspired our work on this topic. Denote

$$
F_{i}(x, \mu):=c^{\mathrm{T}} x+\int_{Z_{i}} f_{i}(z, x) \mu(\mathrm{d} z) \quad(i=1,2) .
$$

Proposition 3.2. For $i=1$ (linear recourse) as well as for $i=2$ (quadratic recourse) the following holds:

Let $B \subset \mathbb{R}^{m}$ nonempty, compact. Let $p \in(1,+\infty)$ and $K \in(0,+\infty)$. Fix some $\mu \in \mathscr{P}\left(Z_{i} ; p, K\right)$. Then there exists $L_{F_{i}}>0$ such that

$$
\left|F_{i}(x, \mu)-F_{i}(y, \nu)\right| \leqslant L_{F_{i}}\left(\|x-y\|+\beta(\nu, \mu)^{1-1 / p}\right)
$$

for all $x, y \in B$ and all $\nu \in \mathscr{P}\left(Z_{i} ; p, K\right)$.

Proof. We have for $i=1,2$,

$$
\left|F_{i}(x, \mu)-F_{i}(y, \nu)\right| \leqslant\left|F_{i}(x, \mu)-F_{i}(y, \mu)\right|+\left|F_{i}(y, \mu)-F_{i}(y, \nu)\right| .
$$


To see that there exist $\tilde{L}_{i}>0(i=1,2)$ such that the first term on the right-hand side is bounded above on $B$ by $\tilde{L}_{i}\|x-y\|$ recall from parametric programming the well-known fact that the functions $f_{i}(i=1,2)$ are convex with respect to $x$. Therefore $F_{i}(\cdot, \mu)(i=1,2)$ are Lipschitzian on compact sets.

Concerning the second term we have for the linear recourse case by Theorem 3.3 in [49] that there exists $\hat{L}_{1}>0$ (independent of $y$ ) such that

$$
\left|F_{1}(y, \mu)-F_{1}(y, \nu)\right| \leqslant \hat{L}_{1} \beta(\nu, \mu)^{1-1 / p}
$$

for all $y \in B$ and all $\nu \in \mathscr{P}\left(Z_{1} ; p, K\right)$. This completes the proof for $i=1$.

We continue the proof for quadratic recourse. To this end let

$$
\tilde{\varphi}(b, d):=\sup _{v}\left\{-\frac{1}{2} v^{\mathrm{T}} H v+d^{\mathrm{T}} v: A v \leqslant b, v \geqslant 0\right\}
$$

and denote

$$
\operatorname{dom} \tilde{\varphi}:=\left\{(b, d) \in \mathbb{R}^{r+1}: \tilde{\varphi}(b, d) \in \mathbb{R}\right\} .
$$

In view of the Theorems 5.5.1, 5.5.2 and Note 5.5.5 in [4] we have that $\tilde{\varphi}$ is continuous on $\operatorname{dom} \tilde{\varphi}$ and that there exist a positive integer $N, M_{j} \subset \mathbb{R}^{r+l}, D_{j} \in L\left(\mathbb{R}^{r+l}, \mathbb{R}^{r+l}\right)$ $(j=1, \ldots, N)$ such that $\left\{\mathcal{M}_{j}: j=1, \ldots, N\right\}$ is a partition of dom $\tilde{\varphi}$ and

$$
\tilde{\varphi}(b, d)=\left(D_{j}(b, d)\right)^{\mathrm{T}}(b, d) \quad \text { if }(b, d) \in \mathcal{M}_{j} .
$$

Now, put

$$
\hat{K}:=\max _{j=1, \ldots, N}\left\|D_{j}\right\|
$$

and let $\bar{r}>0$. Then, for all $j=1, \ldots, N, \tilde{\varphi}$ is Lipschitzian with modulus $\left\|D_{j}\right\| \cdot \bar{r}$ on

$$
\mathcal{M}_{j} \cap\left\{(b, d) \in \mathbb{R}^{r+l}:\|b\|+\|d\| \leqslant \bar{r}\right\} .
$$

Using [26, Theorem 2.1] one shows analogously to [13, Theorem 2.2] that $\tilde{\varphi}$ is Lipschitzian with modulus $\hat{K} \cdot \bar{r}$ on

$$
\operatorname{dom} \tilde{\varphi} \cap\left\{(b, d) \in \mathbb{R}^{r+l}:\|b\|+\|d\| \leqslant \bar{r}\right\} .
$$

The remainder of the proof parallels the proof of Theorem 3.3 in [49].

For arbitrary $y \in B, z=(b, a, T), \tilde{z}=(\tilde{b}, \tilde{a}, \tilde{T})$ we have

$$
\begin{aligned}
& \left|f_{2}(z, y)-f_{2}(\tilde{z}, y)\right| \\
& \quad=|\tilde{\varphi}(b, a-T y)-\tilde{\varphi}(\tilde{b}, \tilde{a}-\tilde{T} y)| \\
& \quad \leqslant \hat{K} \max \{\|b\|+\|a-T y\|,\|\tilde{b}\|+\|\tilde{a}-\tilde{T} y\|\}(\|b-\tilde{b}\|+\|a-\tilde{a}+(\tilde{T}-T) y\|) \\
& \quad \leqslant \tilde{K} \max \{\|z\|,\|\tilde{z}\|\}\|z-\tilde{z}\|
\end{aligned}
$$

with a suitable constant $\tilde{K}>0$. (Note that $B$ is bounded.) Hence, for each $y \in B$, $g_{2}:=f_{2}(\cdot, y): Z_{2} \rightarrow \mathbb{R}$ fulfils the Lipschitz condition (3.1) with $L(t):=L_{0} \cdot t, t \in \mathbb{R}^{+}$ 
and $L_{0}:=\tilde{K}$. Now, we obtain from Theorem 3.1 that there exists $\hat{L}_{2}>0$ (independent of $y$ ) such that

$$
\left|\int_{Z_{2}} f_{2}(z, y) \mu(\mathrm{d} z)-\int_{Z_{2}} f_{2}(z, y) \nu(\mathrm{d} z)\right| \leqslant \hat{L}_{2} \beta(\nu, \mu)^{1-1 / p}
$$

for all $y \in B$ and all $\nu \in \mathscr{P}\left(Z_{2} ; p, K\right)$. Putting $L_{F_{2}}:=\max \left\{\tilde{L}_{2}, \hat{L}_{2}\right\}$ we complete the proof.

We end this section by studying the consequences of Proposition 3.2 and Theorem 2.5 for distribution sensitivity of stochastic programs with linear and quadratic recourse, respectively.

Let $p \in(1,+\infty)$ and $K \in(0,+\infty)$. The sets $\mathscr{P}\left(Z_{i} ; p, K\right)(i=1,2)$ equipped with the metric $\beta$ serve as parameter spaces. Since for any fixed parameter $\mu$ the programs (3.3) and (3.4) are convex (recall the convexity argument given in the preceding proof), there is no distinction between locally and globally optimal values (and local and global minimizers, respectively). Hence let us denote by $\varphi_{i}$ and $\psi_{i}(i=1,2)$ the mappings assigning to each parameter the optimal value and the set of optimal solutions, respectively (of (3.3) and (3.4), respectively).

Theorem 3.3. For $i=1$ (linear recourse) as well as for $i=2$ (quadratic recourse) the following holds:

Consider (3.3) and (3.4) with the same general assumptions as above. Fix some $\mu \in \mathscr{P}\left(Z_{i} ; p, K\right)$ and assume that $\psi_{i}(\mu)$ is nonempty and bounded. Then we have that $\psi_{i}$ is u.s.c. at $\mu$ with respect to the metric space $\left(\mathscr{P}\left(Z_{i} ; p, K\right), \beta\right)$ and there exist constants $\delta_{\varphi_{i}}>0$ and $L_{\varphi_{i}}>0$ such that

$$
\psi_{i}(\nu) \neq \emptyset \quad \text { and }\left|\varphi_{i}(\nu)-\varphi_{i}(\mu)\right| \leqslant L_{\varphi_{i}} \beta(\nu, \mu)^{1-1 / p}
$$

whenever $\nu \in \mathscr{P}\left(Z_{i} ; p, K\right)$ and $\beta(\nu, \mu)<\delta_{\varphi_{i}}$.

Proof. We check the hypotheses of Theorem 2.5. The metric space $(T, d)$ is given by $\left(\mathscr{P}\left(Z_{i} ; p, K\right), \beta\right)(i=1,2)$. As (nonempty) sets of global solutions $\psi_{i}(\mu)(i=1,2)$ are CLM sets, boundedness of $\psi_{i}(\mu)$ implies hypothesis (i) of Theorem 2.5. Hypotheses (ii) and (iii) of Theorem 2.5 trivially hold and (iv) is just the assertion of Proposition 3.2 .

If we restrict the parameter space to a tight subset of $\mathscr{P}\left(Z_{1} ; p, K\right)$ then, for the SLP-case, qualitative stability with respect to weak convergence of probability measures (i.e. continuity of $\varphi_{1}$ and upper semicontinuity of $\psi_{1}$ ) is already implied by Theorems 2.2 and 3.2 in [46].

We continue with applying Theorem 3.3 in the situation where $\mu$ is incompletely known and estimated by empirical measures, thus giving an idea of how to derive asymptotic statistical properties for optimal values of stochastic programs. 
Let $\mu \in \mathscr{P}\left(Z_{i}\right)(i=1,2)$ and $\left(z_{j}\right)_{j \in \mathbb{N}}$ be a sequence of independent random variables on a probability space $(\Omega, \mathscr{A}, P)$ with values in $Z_{i}$ and common distribution $\mu$. Then the empirical measures are given by

$$
\mu_{n}(\omega):=n^{-1} \sum_{j=1}^{n} \delta_{z_{j}(\omega)} \quad(\omega \in \Omega, n \in \mathbb{N}) .
$$

(Here $\delta_{z}$ denotes the measure with unit mass at $z$.)

Corollary 3.4. Assume the hypotheses of Theorem 3.3, and let $C$ be bounded. Then for $i=1,2$ there exists a constant $K_{i}>0$ such that

$$
E\left[\left|\varphi_{i}(\mu)-\varphi_{i}\left(\mu_{n}(\omega)\right)\right|\right] \leqslant K_{i}\left(E\left[\beta\left(\mu, \mu_{n}(\omega)\right)\right]\right)^{1-1 / p} \quad(n \in \mathbb{N}) .
$$

(Here $E[\cdot]$ denotes the mean value with respect to $P$.)

Proof. Let $i \in\{1,2\}$. Then we have from Theorem 3.3 that there exist constants $\delta_{i}>0, L_{i}>0$ such that

$$
\left|\varphi_{i}(\mu)-\varphi_{i}(\nu)\right| \leqslant L_{i} \beta(\mu, \nu)^{1-1 / p}
$$

whenever $\nu \in \mathscr{P}\left(Z_{i} ; p, K\right)$ and $\beta(\mu, \nu)<\delta_{i}$. Note that the Hölder modulus $L_{i}$ of $\varphi_{i}$ has the special structure

$$
L_{i}=\tilde{K}_{i}\left[1+\left(\int_{Z_{i}}\|z\|^{2 p} \nu(\mathrm{d} z)\right)^{1 / p}\right]
$$

with some constant $\tilde{K}_{i}>0$ (Remark 2.7., Theorems 3.1 and 3.3). Hence, for fixed $n \in \mathbb{N}$, there holds for all $\omega \in A_{i}:=\left\{\omega \in \Omega: \beta\left(\mu, \mu_{n}(\omega)\right)<\delta_{i}\right\}$,

$$
\left|\varphi_{i}(\mu)-\varphi_{i}\left(\mu_{n}(\omega)\right)\right| \leqslant K\left[1+\left(n^{-1} \sum_{j=1}^{n}\left\|z_{j}(\omega)\right\|^{2 p}\right)^{1 / p}\right] \beta\left(\mu, \mu_{n}(\omega)\right)^{1-1 / p}
$$

and we obtain applying Hölder's and Chebyshev's inequalities

$$
\begin{aligned}
E[ & \left.\left|\varphi_{i}(\mu)-\varphi_{i}\left(\mu_{n}(\omega)\right)\right|\right] \\
= & \int_{A_{i}}\left|\varphi_{i}(\mu)-\varphi_{i}\left(\mu_{n}(\omega)\right)\right| \mathrm{d} P+\int_{\Omega \backslash A_{i}}\left|\varphi_{i}(\mu)-\varphi_{i}\left(\mu_{n}(\omega)\right)\right| \mathrm{d} P \\
\leqslant & K\left[1+\left(n^{-1} \sum_{j=1}^{n} E\left[\left\|z_{j}(\omega)\right\|^{2 p}\right]\right)^{1 / p}\right]\left(E\left[\beta\left(\mu, \mu_{n}(\omega)\right)\right]\right)^{1-1 / p} \\
& +P\left(\Omega \backslash A_{i}\right)^{1-1 / p}\left(E\left[\left|\varphi_{i}(\mu)-\varphi_{i}\left(\mu_{n}(\omega)\right)\right|^{p}\right]\right)^{1 / p} \\
\leqslant & \left\{K\left[1+\left(\int_{Z_{i}}\|z\|^{2 p} \mu(\mathrm{d} z)\right)^{1 / p}\right]\right. \\
& \left.+\frac{1}{\delta_{i}}\left(\delta_{i} E\left[\left|\varphi_{i}(\mu)-\varphi_{i}\left(\mu_{n}(\omega)\right)\right|^{p}\right]\right)^{1 / p}\right\}\left(E\left[\beta\left(\mu, \mu_{n}(\omega)\right)\right]\right)^{1-1 / p} .
\end{aligned}
$$


Since $C$ is bounded, we have for each $\nu \in \mathscr{P}\left(Z_{i}\right)$ that

$$
\left|\varphi_{i}(\nu)\right| \leqslant \max _{x \in C}\left\{\left|c^{\mathrm{T}} x\right|+\int_{Z_{i}}\left|f_{i}(z, x)\right| \nu(\mathrm{d} z)\right\},
$$

and in view of (3.5),

$$
\left|\varphi_{i}(\nu)\right|^{p} \leqslant \hat{K}_{i}\left[1+\left(\int_{Z_{i}}\|z\|^{2 p} \nu(\mathrm{d} z)\right)^{1 / p}\right]
$$

with some constant $\hat{K}_{i}>0$. Therefore, $E\left[\left|\varphi_{i}(\mu)-\varphi_{i}\left(\mu_{n}(\omega)\right)\right|^{p}\right]$ is bounded with respect to $n \in \mathbb{N}$ and the assertion is proved.

Note that there exist (sharp) bounds for $E\left[\beta\left(\mu, \mu_{n}(\omega)\right)\right]$ of the type $c n^{-1 / k}$ with constants $c>0$ and $k>2$ [14]. Concerning $k$ we remark that it is bounded below by the dimension of the (smallest) Euclidean space including the support of $\mu$ and that it depends on the existence of certain moments of $\mu$ (see [14] for details).

\section{Stability of solutions to recourse problems}

Now we study consequences of Theorem 2.6 with respect to quantitative stability of optimal solutions to recourse problems. Having in mind Proposition 3.2, an inspection of Theorem 2.6 indicates the central role of the concept of a strict local minimizer of some order $q \in[1,+\infty)$ for quantifying upper semicontinuity of the optimal set-mapping $\psi$. In what follows we emphasize the case when $q=2$.

It is well known (cf., e.g., [44, Theorem 2.2]) that for the standard nonlinear programming problem with inequality and equality constraints each point satisfying the Kuhn-Tucker necessary optimality conditions as well as the sufficient second order condition [44, Definition 2.1] is a strict local minimizer of order 2. Of course, this argumentation needs twice differentiability of the problem data, although there exist generalizations to the locally Lipschitzian case (e.g. [3]). In the sensitivity analysis that follows, however, we restrict ourselves to the case where the original problem is twice differentiable. In view of the convexity of the problem and the polyhedrality of its constraint set, the above sufficient condition for the existence of a strict local (therefore global) minimizer of order 2 reduces considerably. It already holds if there exists an optimal solution where the Hessian of the objective is non-singular. In the sequel, this will be the key assumption to be verified.

Let us consider the following program with linear recourse

$$
\min _{y}\left\{c^{\mathrm{T}} y+\int_{Z} f(y, z) \mu(\mathrm{d} z): y \in C\right\}
$$

where $C \subset \mathbb{R}^{m}$ is a nonempty convex polyhedron and $c \in \mathbb{R}^{m}$,

$$
f(y, z):=\min _{v}\left\{q^{\mathbf{T}} v:[D,-D] v=z-y, v \geqslant 0\right\},
$$


$Z=\mathbb{R}^{m}, D \in L\left(\mathbb{R}^{m}, \mathbb{R}^{m}\right)$ nonsingular and $\mu \in \mathscr{P}(Z)$. For convenience we denote the objective in (4.1) by $F(y, \mu)$ and split the vector $q \in \mathbb{R}^{2 m}$ into $q^{+} \in \mathbb{R}^{m}$ and $q^{-} \in \mathbb{R}^{m}$ consisting of the first and the last $m$ components of $q$, respectively.

We remark that the above class of problems was brought to our attention by the paper [9] of Birge and Wets, where such problems serve as approximations to more general recourse problems.

Proposition 4.1. Consider (4.1) with the above assumptions, let $q^{+}+q^{-}>0$ and suppose that for the one-dimensional marginal distributions $\tilde{\mu}_{i}$ of $\tilde{\mu}:=\mu \circ D(i=1, \ldots, m)$ there exist densities $\tilde{\theta}_{i}$ which are on int supp $\tilde{\mu}_{i}$ both continuous and strictly positive. Then $\nabla_{y y} F(y, \mu)$ exists and is nonsingular for each $y \in$ int $\operatorname{supp} \mu$. ("supp $\nu$ " denotes the support of the measure $\nu$.)

Proof. The function $f(y, z)$ given by (4.2) is finite everywhere, since one always finds a feasible $v$ and since by $q^{+}+q^{-}>0$ the constraint set of the dual to the problem defining $f$ is nonempty.

Following [9] we have the expression

$$
f(y, z)=\sum_{i=1}^{m} f_{i}(y, z)
$$

where

$$
\begin{aligned}
& f_{i}(y, z)= \begin{cases}q_{D(i)}^{+}\left(D^{-1}\right)_{i} \cdot(z-y) & \text { if }\left(D^{-1}\right)_{i} \cdot(z-y) \geqslant 0, \\
q_{D(i)}^{-}\left(D^{-1}\right)_{i} \cdot(y-z) & \text { if }\left(D^{-1}\right)_{i \cdot}(z-y)<0,\end{cases} \\
& q_{D(i)}^{+}:=\min \left\{q^{\mathrm{T}} v:[D,-D] v=D(i), v \geqslant 0\right\}, \\
& q_{D(i)}^{-}:=\min \left\{q^{\mathrm{T}} v:[D,-D] v=-D(i), v \geqslant 0\right\} .
\end{aligned}
$$

$\left(D^{-1}\right)_{i}$. denotes the $i$ th row of $D^{-1}, D(i)$ the $i$ th column of $D$. By inspection of the dual problems to (4.3) and (4.4) one confirms

$$
q_{D(i)}^{+}=q_{i}^{+} \quad \text { and } \quad q_{D(i)}^{-}=q_{i}^{-} .
$$

Now

$$
F(y, \mu)=c^{\mathrm{T}} y+\sum_{i=1}^{m} F_{i}(y, \mu):=c^{\mathrm{T}} y+\sum_{i=1}^{m} \int_{Z} f_{i}(y, z) \mu(\mathrm{d} z) .
$$

Using the transformation $\tilde{z}=D^{-1} z$ the last expression becomes

$$
c^{\mathrm{T}} y+\sum_{i=1}^{m} \int_{\mathcal{Z}} f_{i}(y, D \tilde{z})|\operatorname{det} D| \tilde{\mu}(\mathrm{d} \tilde{z}) .
$$

Observe that

$$
f_{i}(y, D \tilde{z})= \begin{cases}q_{i}^{+}\left(\tilde{z}_{i}-\left(D^{-1}\right)_{i} \cdot y\right) & \text { if } \tilde{z_{i}} \geqslant\left(D^{-1}\right)_{i} \cdot y, \\ q_{i}^{-}\left(\left(D^{-1}\right)_{i} \cdot y-\tilde{z}_{i}\right) & \text { if } \tilde{z}_{i}<\left(D^{-1}\right)_{i} \cdot y .\end{cases}
$$


Therefore

$$
\begin{aligned}
& F_{i}(y, \mu)=|\operatorname{det} D| \cdot\left\{q_{i}^{+} \int_{\mathbb{R}} t \tilde{\theta}_{i}(t) \mathrm{d} t-q_{i}^{+}\left(D^{-1}\right)_{i} \cdot y\right. \\
&\left.-\left(q_{i}^{+}+q_{i}^{-}\right) \int_{-\infty}^{\left(D^{-1}\right)_{i} \cdot y}\left(t-\left(D^{-1}\right)_{i} \cdot y\right) \tilde{\theta}_{i}(t) \mathrm{d} t\right\},
\end{aligned}
$$

and we have

$$
\nabla_{y} F_{i}(y, \mu)=|\operatorname{det} D| \cdot\left(-q_{i}^{+}+\left(q_{i}^{+}+q_{i}^{-}\right) \int_{-\infty}^{\left(D^{-1}\right)_{i}, y} \tilde{\theta}_{i}(t) \mathrm{d} t\right)\left(D^{-1}\right)_{i}^{\mathrm{T}} .
$$

Hence

$$
\nabla_{y y} F_{i}(y, \mu)=|\operatorname{det} D|\left(q_{i}^{+}+q_{i}^{-}\right) \tilde{\theta}_{i}\left(\left(D^{-1}\right)_{i} y\right)\left(D^{-1}\right)_{i \cdot}^{\mathrm{T}}\left(D^{-1}\right)_{i \cdot \cdot}
$$

(Note that the existence of $\nabla_{y y} F_{i}(y, \mu)$ is ensured for $y \in$ int supp $\mu$ since $y \in$ int supp $\mu$ implies $\left(D^{-1}\right)_{i} . y \in$ int supp $\tilde{\mu}_{i}$.) Our suppositions now imply

$$
|\operatorname{det} D|\left(q_{i}^{+}+q_{i}^{-}\right) \tilde{\theta}_{i}\left(\left(D^{-1}\right)_{i} \cdot y\right)>0 \text { for } i=1, \ldots, m \text {. }
$$

Hence

$$
\xi^{\mathrm{T}} \nabla_{y y} F_{i}(y, \mu) \xi \geqslant 0 \quad(i=1, \ldots, m)
$$

where equality holds only for those $\xi$ which satisfy $\left(D^{-1}\right)_{i} \xi=0$. Since the rows of $D^{-1}$ are independent we obtain

$$
\xi^{\mathrm{T}} \nabla_{y y} F(y, \mu) \xi>0
$$

for all $\xi \in \mathbb{R}^{m}, \xi \neq 0$ and the assertion is verified.

Remark 4.2. If supp $\mu=\mathbb{R}^{m}$ then the restriction on $y$ in the assertion of the above proposition can be dropped. In general, however this is not possible as is illustrated by the function $F\left(\cdot, \mu_{0}\right)$ in Example 2.1. This function does not possess a Hessian at the boundary of the support and has the Hessian 0 outside the support.

Now we are going to apply Theorem 2.6 to problem (4.1) understood as a parametric programming problem with respect to the underlying probability measure. As in the previous section, let $p \in(1,+\infty)$ and $K \in(0,+\infty)$ to fix a parameter set $\mathscr{P}(Z ; p, K)$.

Theorem 4.3. Consider problem (4.1) with general assumptions as above, let $q^{+}+q^{-}>0$ and assume that for some fixed $\mu \in \mathscr{P}(Z ; p, K)$ (representing the unperturbed problem) the set $\psi(\mu)$ is nonempty and belongs to int supp $\mu$. Suppose further that for the one-dimensional marginal distributions $\tilde{\mu}_{i}$ of $\tilde{\mu}:=\mu \circ D(i=1, \ldots, m)$ there exist densities $\tilde{\theta}_{i}$ which are both continuous and strictly positive on int supp $\tilde{\mu}_{i}$. Then $\psi(\mu)$ is a singleton $(\operatorname{say} \psi(\mu)=\{\bar{y}\})$ and there exist $L_{\psi}^{1}>0$ and $\eta_{\psi}^{1}>0$ such that we have for any $\nu \in \mathscr{P}(Z ; p, K)$ for which $\beta(\mu, \nu)<\eta_{\psi}^{1}$ that

$$
\psi(\nu) \neq \emptyset \text { and }\|\bar{y}-y\| \leqslant L_{\psi}^{1} \beta(\mu, \nu)^{(1-1 / p) / 2} \quad \text { for all } y \in \psi(\nu) .
$$


Proof. In view of Proposition 4.1 and the argumentation at the beginning of this section the unperturbed problem has a strict local minimizer of order 2 . Hence $\psi(\mu)$ is a singleton and hypothesis (i) of Theorem 2.6 holds. Hypothesis (iv) is implied by Proposition 3.2 whereas the remaining hypotheses of Theorem 2.6 trivially hold. Theorem 2.6 now completes the proof.

Next, we extend the above stability result to the more general class of problems given by

$$
\min _{x}\left\{c^{\mathrm{T}} x+\int_{Z} f(x, z) \mu(\mathrm{d} z): x \in C\right\}
$$

where $C \subset \mathbb{R}^{m}$ is a nonempty convex polyhedron and $c \in \mathbb{R}^{m}$,

$$
f(x, z):=\min _{v}\left\{q^{\mathrm{T}} v:[D,-D] v=z-T x, v \geqslant 0\right\},
$$

$Z=\mathbb{R}^{r}, D \in L\left(\mathbb{R}^{r}, \mathbb{R}^{r}\right)$ nonsingular, $T \in L\left(\mathbb{R}^{m}, \mathbb{R}^{r}\right)$ with full rank and $\mu \in \mathscr{P}(Z)$. As in (4.1) we split $q$ into $q^{+}$and $q^{-}$. Unless $m=r$, a solution to (4.5), if it exists, cannot always be ensured to be uniquely determined. Hence, in general there is not much hope of identifying strict local minimizers of any order, and Theorem 2.6 cannot be applied directly. However, under an additional assumption on the linear part of the objective in (4.5) we can show the assertion of Theorem 4.3 to hold with little modification.

Theorem 4.4. Consider (4.5) with general assumptions as above and let the vector $c \in \mathbb{R}^{m}$ admit a representation $c^{\mathrm{T}}=\tilde{c}^{\mathrm{T}} T$ with some $\tilde{c} \in \mathbb{R}^{r}$. Suppose further $q^{+}+q^{-}>0$ and that for the one-dimensional marginal distributions $\tilde{\mu}_{i}$ of $\tilde{\mu}:=\mu \circ D(i=1, \ldots, r)$ there exist densities $\tilde{\theta}_{i}$ which are both continuous and strictly positive on int supp $\tilde{\mu}_{i}$. Assume that for some fixed $\mu \in \mathscr{P}(Z ; p, K)$ the set $\psi(\mu)$ is nonempty and that we have $T x \in$ int supp $\mu$ for any $x \in \psi(\mu)$. Then there exist $L_{\psi}^{2}>0$ and $\eta_{\psi}^{2}>0$ such that we have for any $\nu \in \mathscr{P}(Z ; p, K)$ for which $\beta(\mu, \nu)<\eta_{\psi}^{2}$ that

$$
\psi(\nu) \neq \emptyset \quad \text { and } \quad d_{\mathrm{H}}(\psi(\nu), \psi(\mu)) \leqslant L_{\psi}^{2} \beta(\nu, \mu)^{(1-1 / p) / 2}
$$

where $d_{\mathrm{H}}$ denotes the Hausdorff distance.

Proof. Denote $T(C):=\left\{y \in \mathbb{R}^{r}: y=T x, x \in C\right\}$ and consider

$$
\min _{y}\left\{\tilde{c}^{\mathrm{T}} y+\int_{z} \tilde{f}(y, z) \nu(\mathrm{d} z): y \in T(C)\right\},
$$

where

$$
\tilde{f}(y, z):=\min _{v}\left\{q^{\mathrm{T}} v:[D,-D] v=z-y, v \geqslant 0\right\} .
$$


Since $T(C)$ is again a nonempty convex polyhedron (4.6) fits into (4.1). Let $\tilde{\psi}(\nu)$ denote the solution set of (4.6) and put

$$
C_{y}:=\left\{x \in \mathbb{R}^{m}: y=T x, x \in C\right\} .
$$

Using the representation of $c^{\mathrm{T}}$ one then confirms

$$
\psi(\nu)=\bigcup_{y \in \tilde{\psi}(\nu)} C_{y} .
$$

Theorem 4.3 now yields that there exists $\eta_{\bar{\psi}}^{1}>0$ such that $\nu \in \mathscr{P}(Z ; p, K)$ and $\beta(\nu, \mu)<\eta_{\tilde{\psi}}^{1}$ imply $\tilde{\psi}(\nu) \neq \emptyset$. Note that for any $y \in \tilde{\psi}(\nu)$ we have $C_{y} \neq \emptyset$ and hence by $(4.7)$,

$$
\psi(\nu) \neq \emptyset \text {. }
$$

Now observe that by Theorem 4.3, $\tilde{\psi}(\mu)$ is a singleton (let $\tilde{\psi}(\mu)=\{\bar{y}\}) ;(4.7)$ implies $\psi(\mu)=C_{\bar{y}}$. Furthermore, we have by (4.7),

$$
d_{\mathrm{H}}(\psi(\mu), \psi(\nu)) \leqslant \sup _{y \in \tilde{\psi}(\nu)} d_{\mathrm{H}}\left(C_{\bar{y}}, C_{y}\right) .
$$

By Hoffman's theorem (cf. [43, p. 760]) there exists $L_{\mathrm{H}}>0$ which is independent of $y$ such that

$$
d_{\mathrm{H}}\left(C_{\bar{y}}, C_{y}\right) \leqslant L_{\mathrm{H}} \cdot\|\bar{y}-y\| .
$$

Hence

$$
d_{\mathrm{H}}(\psi(\mu), \psi(\nu)) \leqslant L_{\mathrm{H}} \cdot \sup _{y \in \tilde{\psi}(\nu)}\|\bar{y}-y\|
$$

and in view of Theorem 4.3 the right-hand side of the last inequality can be estimated above by

$$
L_{\mathrm{H}} \cdot L_{\tilde{\psi}}^{1} \beta(\nu, \mu)^{(1-1 / p) / 2} .
$$

Remark 4.5. In Theorems 4.3 and 4.4, the estimates on the distance of the solution sets hold with exponent $\frac{1}{2}$ on the right-hand side, if there exists a compact set containing all the supports of the involved probability measures.

To show that the exponent on the right-hand sides in the estimates in the Theorems 4.3 and 4.4 is the best possible we give the following example.

Example 4.6. Let $F(x, \mu)$ and $\mu_{0}$ be as in Example 2.1, let $\mu_{n}(n \in \mathbb{N})$ be given by the distribution function

$$
D_{n}(s)= \begin{cases}0, & s<-\frac{1}{2}, \\ s+\frac{1}{2}, & -\frac{1}{2} \leqslant s<-1 / \sqrt{n}, \\ \frac{1}{2}, & -1 / \sqrt{n} \leqslant s<1 / \sqrt{n}, \\ s+\frac{1}{2}, & 1 / \sqrt{n} \leqslant s<\frac{1}{2}, \\ 1, & \frac{1}{2} \leqslant s .\end{cases}
$$

Let $P(\mu)=\min \{F(x, \mu): x \in \mathbb{R}\}$. Then $\psi\left(\mu_{0}\right)=\{0\}, \psi\left(\mu_{n}\right)=[-1 / \sqrt{n}, 1 / \sqrt{n}]$ and $\beta\left(\mu_{n}, \mu_{0}\right) \leqslant 4 / n$. Now, we have $d_{\mathrm{H}}\left(\psi\left(\mu_{n}\right), \psi\left(\mu_{0}\right)\right)=1 / \sqrt{n}$, i.e. the exponent $\frac{1}{2}$ which is obtained from Theorem 4.3 in view of Remark 4.5 cannot be increased. 
Now we turn our attention to stochastic programs with quadratic recourse. We will study quantitative stability of optimal solutions when the recourse problem has box-diagonal structure.

Consider

$$
\min _{y}\left\{c^{\mathrm{T}} y+\int_{z} f(y, z) \mu(\mathrm{d} z): y \in C\right\}
$$

where $C \subset \mathbb{R}^{m}$ is a nonempty convex polyhedron and $c \in \mathbb{R}^{m}$,

$$
f(y, z):=\max _{v}\left\{-\frac{1}{2} v^{\mathrm{T}} \tilde{D} v+(z-y)^{\mathrm{T}} v: a \leqslant v \leqslant b\right\},
$$

$Z=\mathbb{R}^{m}, a, b \in \mathbb{R}^{m}(a \leqslant b), \tilde{D} \in L\left(\mathbb{R}^{m}, \mathbb{R}^{m}\right)$ diagonal with positive elements $d_{i}(i=$ $1, \ldots, m), \mu \in \mathscr{P}(Z)$. We denote the objective in (4.8) by $F(y, \mu)$.

Proposition 4.7. Suppose in (4.8) that for the one-dimensional marginal distributions $\mu_{i}$ of $\mu(i=1, \ldots, m)$ there exist densities $\theta_{i}$ which are both continuous and strictly positive on int supp $\mu_{i}$. Then $\nabla_{y y} F(y, \mu)$ exists and is nonsingular for all $y \in \mathbb{R}^{m}$ such that

$$
\left\{s \in \mathbb{R}^{m}: \tilde{D} a+y \leqslant s \leqslant \tilde{D} b+y\right\} \subset \text { int } \operatorname{supp} \mu .
$$

Proof. We find that $f(y, z)$ is separable, namely

$$
f(y, z)=\sum_{i=1}^{m} f_{i}\left(y_{i}, z_{i}\right)
$$

where

$$
f_{i}\left(y_{i}, z_{i}\right)= \begin{cases}\left(1 /\left(2 d_{i}\right)\left(z_{i}-y_{i}\right)^{2},\right. & \text { if } z_{i}-y_{i} \in\left[a_{i} d_{i}, b_{i} d_{i}\right], \\ a_{i}\left(z_{i}-y_{i}\right)-\frac{1}{2} d_{i} a_{i}^{2}, & \text { if } z_{i}-y_{i}<a_{i} d_{i}, \\ b_{i}\left(z_{i}-y_{i}\right)-\frac{1}{2} d_{i} b_{i}^{2}, & \text { if } z_{i}-y_{i}>b_{i} d_{i},\end{cases}
$$

for $i=1, \ldots, m$. Denote

$$
F_{i}\left(y_{i}\right):=\int f_{i}\left(y_{i}, z_{i}\right) \mu_{i}\left(\mathrm{~d} z_{i}\right)
$$

where " $\mu_{i}\left(\mathrm{~d} z_{i}\right)$ " indicates that the integral is taken with respect to the marginal distribution. If the second derivatives $\left(\mathrm{d}^{2} / \mathrm{d} y_{i}^{2}\right) F_{i}\left(y_{i}\right)$ exist for $i=1, \ldots, m$, then $\nabla_{y y} F(y, \mu)$ is a diagonal matrix with elements $\left(\mathrm{d}^{2} / \mathrm{d} y_{i}^{2}\right) F_{i}\left(y_{i}\right)$ on the main diagonal. Hence it remains to show that these derivatives exist and that they are positive. We have

$$
\begin{aligned}
F_{i}\left(y_{i}\right)= & \int_{a_{i} d_{i}+y_{i}}^{b_{i} d_{i}+y_{t}} \frac{1}{2 d_{i}}\left(t-y_{i}\right)^{2} \theta_{i}(t) \mathrm{d} t \\
& +\int_{-\infty}^{a_{i} d_{i}+y_{i}}\left[a_{i}\left(t-y_{i}\right)-\frac{1}{2} d_{i} a_{i}^{2}\right] \theta_{i}(t) \mathrm{d} t \\
& +\int_{b_{i} d_{i}+y_{i}}^{\infty}\left[b_{i}\left(t-y_{i}\right)-\frac{1}{2} d_{i} b_{i}^{2}\right] \theta_{i}(t) \mathrm{d} t .
\end{aligned}
$$


In view of condition (4.9) now $\left(\mathrm{d} / \mathrm{d} y_{i}\right) F_{i}\left(y_{i}\right)$ exists and we obtain by direct computation

$$
\begin{aligned}
\frac{\mathrm{d}}{\mathrm{d} y_{i}} F_{i}\left(y_{i}\right)= & -\frac{1}{d_{i}} \int_{a_{i} d_{i}+y_{i}}^{b_{i} d_{i}+y_{i}} t \theta_{i}(t) \mathrm{d} t+\frac{1}{d_{i}} y_{i} \int_{a_{i} d_{i}+y_{i}}^{b_{i} d_{i}+y_{i}} \theta_{i}(t) \mathrm{d} t \\
& -a_{i} \int_{-\infty}^{a_{i} d_{i}+y_{i}} \theta_{i}(t) \mathrm{d} t-b_{i} \int_{b_{i} d_{i}+y_{i}}^{\infty} \theta_{i}(t) \mathrm{d} t,
\end{aligned}
$$

and finally

$$
\frac{\mathrm{d}^{2}}{\mathrm{~d} y_{i}^{2}} F_{i}\left(y_{i}\right)=\frac{1}{d_{i}} \int_{a_{i} d_{i}+y_{i}}^{b_{i} d_{i}+y_{i}} \theta_{i}(t) \mathrm{d} t .
$$

These expressions are positive for $i=1, \ldots, m$, and the proof is complete.

In analogy to Remark 4.2 we note that, in case supp $\mu=\mathbb{R}^{m}$, condition (4.9) in the above theorem is not a restriction. Let us now view problem (4.8) as a parametric program with respect to the underlying probability measure. With $p \in(1,+\infty)$ and $K \in(0,+\infty)$ we fix the parameter space $\mathscr{P}(Z ; p, K)$ and obtain the following consequence of Theorem 2.6.

Theorem 4.8. Consider (4.8) with general assumptions as above and suppose that for some fixed $\mu \in \mathscr{P}(Z ; p, K)$ the set $\psi(\mu)$ is nonempty and (4.9) holds for all $y \in \psi(\mu)$. Further, assume that for the one-dimensional marginal distributions $\mu_{i}$ of $\mu$ ( $i=$ $1, \ldots, m)$ there exist densities which are both continuous and strictly positive on int supp $\mu_{i}$. Then $\psi(\mu)$ is a singleton (say $\psi(\mu)=\{\bar{y}\}$ ) and there exist $L_{\psi}^{3}>0$ and $\eta_{\psi}^{3}>0$ such that we have for any $\nu \in \mathscr{P}(Z ; p, K)$ for which $\beta(\mu, \nu)<\eta_{\psi}^{3}$ that

$$
\psi(\nu) \neq \emptyset \quad \text { and } \quad\|\bar{y}-y\| \leqslant L_{\psi}^{3} \beta(\mu, \nu)^{(1-1 / p) / 2} \text { for all } y \in \psi(\nu) .
$$

The proof is a repetition of the proof of Theorem 4.3 using Proposition 4.7 instead of Proposition 4.1.

It is possible to generalize Theorem 4.8 in exactly the same way as this has been done for linear recourse in Theorem 4.4. As can be seen from the proofs, the stability results of the present section remain unchanged if one has strict positivity of the densities only on a neighbourhood around some point determined by the optimal solution set of the unperturbed problem.

\section{Stability in chance constrained programming}

In this section we present stability results for (locally) optimal sets and optimal values of chance constrained stochastic programs. The problem we shall consider is

$$
\min \left\{f(x): x \in \mathbb{R}^{m}, \mu\left(\left\{z \in \mathbb{R}^{s}: x \in X(z)\right\}\right) \geqslant p_{0}\right\},
$$


where $f$ is a function from $\mathbb{R}^{m}$ into $\mathbb{R}, X$ is a measurable multifunction from $\mathbb{R}^{s}$ into $\mathbb{R}^{m}, p_{0} \in[0,1]$ and $\mu$ is a Borel probability measure on $\mathbb{R}^{s}$. ( $X$ is measurable iff $\left\{z \in \mathbb{R}^{s}: X(z) \cap A \neq \emptyset\right\} \in \mathscr{B}\left(\mathbb{R}^{s}\right)$ for each closed $A \subset \mathbb{R}^{m}$.)

Since $X$ is measurable, the preimages $X^{-}(x):=\left\{z \in \mathbb{R}^{s}: x \in X(z)\right\}$ are Borel for each $x \in \mathbb{R}^{m}$.

Our approach to stability in the chance constrained case is based on Theorem 2.5. The results we obtain extend those of [49, Section 4], since we consider more general problems (5.1) and we also deal with the stability of local solutions. In order to apply the parametric programming framework (of Section 2), we first have to choose a suitable distance on $\mathscr{P}\left(\mathbb{R}^{s}\right)$. Of course, it again appears to be useful to choose a distance metrizing the topology $\tau_{w}$ of weak convergence on $\mathscr{P}\left(\mathbb{R}^{s}\right)$. But, unfortunately, as turned out in [30], it seems to be difficult to establish general verifiable sufficient conditions to ensure lower semicontinuity of the multifunction

$$
\nu \rightarrow C_{p}(\nu):=\left\{x \in \mathbb{R}^{m}: \nu\left(X^{-}(x)\right) \geqslant p\right\}
$$

considered as a map from $\left(\mathscr{P}\left(\mathbb{R}^{s}\right), \tau_{w}\right)$ to $\mathbb{R}^{m}$. Recall that a multifunction $\Gamma$ from a topological space $(T, \tau)$ to $\mathbb{R}^{m}$ is lower semicontinuous (1.s.c.) at some $t_{0} \in T$ if, for each $x \in \Gamma\left(t_{0}\right)$ and each $\varepsilon>0$, there exists a $\tau$-neighbourhood $U$ of $t_{0}$ such that for each $t \in U, \Gamma(t) \cap B(x, \varepsilon) \neq \emptyset$.

In our first result (which is close to [50, Corollary 3.2.1]) we show that such a verifiable condition can be established if the subset $\mathscr{B}_{0}:=\left\{X^{-}(x): x \in \mathbb{R}^{m}\right\}$ of $\mathscr{B}\left(\mathbb{R}^{s}\right)$ forms a $\mu$-uniformity class. Recall that $\mathscr{B}_{0}$ is a $\mu$-uniformity class if $\sup \{\mid \mu(B)-$ $\left.\mu_{n}(B) \mid: B \in \mathscr{B}_{0}\right\} \rightarrow 0$ as $n \rightarrow \infty$ holds for every sequence $\left(\mu_{n}\right)$ in $\mathscr{P}\left(\mathbb{R}^{s}\right)$ converging weakly to $\mu$ (see e.g. [5,7]).

Proposition 5.1. Let $\mu \in \mathscr{P}\left(\mathbb{R}^{s}\right), p_{0} \in(0,1)$. Assume that $\mathscr{B}_{0}:=\left\{X^{-}(x): x \in \mathbb{R}^{m}\right\}$ is a $\mu$-uniformity class and that the multifunction $p \rightarrow C_{p}(\mu)\left(\right.$ from $\mathbb{R}$ to $\left.\mathbb{R}^{m}\right)$ is l.s.c. at $p_{0}$. Then $\nu \rightarrow C_{p_{0}}(\nu)\left(\right.$ from $\left(\mathscr{P}\left(\mathbb{R}^{s}\right), \tau_{w}\right)$ to $\left.\mathbb{R}^{m}\right)$ is l.s.c. at $\mu$.

Proof. Let $x \in C_{p_{0}}(\mu)$ and $\varepsilon>0$. Then there is a $\delta>0$ such that, for each $p \in \mathbb{R}$ with $\left|p-p_{0}\right|<\delta, C_{p}(\mu) \cap B(x, \varepsilon) \neq \emptyset$. Now we choose a $\tau_{w}$-neighbourhood $U$ of $\mu$ such that $\sup \left\{|\mu(B)-\nu(B)|: B \in \mathscr{B}_{0}\right\}<\delta$ holds for each $\nu \in U$.

Let $\nu \in U$ and put $p:=p_{0}+\sup \left\{|\mu(B)-\nu(B)|: B \in \mathscr{B}_{0}\right\}$. Then we have for each $y \in C_{p}(\mu)$ that

$$
\nu\left(X^{-}(y)\right) \geqslant \mu\left(X^{-}(y)\right)-\left|\mu\left(X^{-}(y)\right)-\nu\left(X^{-}(y)\right)\right| \geqslant p_{0} .
$$

Hence $y \in C_{p_{0}}(\nu)$ and $C_{p_{0}}(\nu) \cap B(x, \varepsilon) \supset C_{p}(\mu) \cap B(x, \varepsilon) \neq \emptyset$. Thus $\nu \mapsto C_{p_{0}}(\nu)$ is 1.s.c. at $\mu$.

Remark 5.2. Proposition 5.1 shows that the multifunction $\nu \mapsto C_{p_{0}}(\nu)$ is 1.s.c. at those measures $\mu \in \mathscr{P}\left(\mathbb{R}^{s}\right)$ which are "smooth" in some sense. The "smoothness" conditions on $\mu$ are connected with the multifunction $X$ (describing the constraints) and with the properties of the mapping $p \mapsto C_{p}(\mu)$ at the prescribed probability level 
$p_{0}$. It is known from [7] that a class $\mathscr{B}_{0}$ of Borel subsets of $\mathbb{R}^{s}$ is a $\mu$-uniformity class if $\lim _{\varepsilon \rightarrow 0} \sup \left\{\mu\left((\partial B)^{\varepsilon}\right): B \in \mathscr{B}_{0}\right\}=0$. (Here $\partial B$ denotes the topological boundary of $B$ and $A^{\varepsilon}$ denotes the $\varepsilon$-enlargement of $A \subset \mathbb{R}^{s}$.)

Let us consider two special classes $\mathscr{B}_{0}$ of Borel sets:

(i) $\mathscr{B}_{\mathbb{R}}:=\left\{(-\infty, z]=X_{i=1}^{s}\left(-\infty, z_{i}\right]: z=\left(z_{1}, \ldots, z_{s}\right)^{\mathrm{T}} \in \mathbb{R}^{s}\right\}$ is a $\mu$-uniformity class for those $\mu \in \mathscr{P}\left(\mathbb{R}^{s}\right)$ whose probability distribution function $F_{\mu}$ is continuous.

(ii) $\mathscr{B}_{C}:=\left\{B \subseteq \mathbb{R}^{s}: B\right.$ is convex and Borel $\}$ is a $\mu$-uniformity class if $\mu$ is absolutely continuous w.r.t. Lebesgue measure on $\mathbb{R}^{s}$ ([5, Theorem 2.11, pp. 22-23]).

Hence, at least, if $\mu$ is absolutely continuous and all preimages $X^{-}(x)\left(x \in \mathbb{R}^{m}\right)$ are convex, Proposition 5.1 appears to be useful (e.g. for qualitative stability results for chance constrained problems). Simple examples show that the lower semicontinuity of $p \mapsto C_{p}(\mu)$ at $p_{0}$ cannot be dispensed with in Proposition 5.1 (cf. also [50, Theorem 3.2]). However, since we are interested in quantitative stability results, we do not pursue this line of research here. Proposition 5.1 indicates that the so-called "variational (or uniform) distance" (which is sometimes also called " $\mathscr{B}$ discrepancy" e.g. in [22]) $\alpha_{\mathscr{B}}(\mu, \nu)\left(\mu, \nu \in \mathscr{P}\left(\mathbb{R}^{s}\right)\right)$ (see (1.2)) might be a suitable (pseudo-) metric on $\mathscr{P}\left(\mathbb{R}^{s}\right)$ for our purposes. Note that $\alpha_{\mathscr{B}_{\mathbb{R}}}$ is the well-known "Kolmogorov distance" on $\mathscr{P}\left(\mathbb{R}^{s}\right)$. Indeed, it turns out in the following that variational distances $\boldsymbol{\alpha}_{\mathscr{B}}$, where $\mathscr{B}$ contains the set $\left\{X^{-}(x): x \in \mathbb{R}^{m}\right\}$, are suitable for the application of the concepts of Section 2.

As a quantitative analogue to Proposition 5.1 our next result asserts a Lipschitz property of the multifunction $\nu \mapsto C_{p_{0}}(\nu)$ at $\mu \in \mathscr{P}\left(\mathbb{R}^{s}\right)$ which is needed for the application of Theorem 2.5. To state the result we need the following Lipschitz property of multifunctions which was introduced in [2] (see also [47]).

A multifunction $\Gamma$ from $\mathbb{R}^{d}$ into $\mathbb{R}^{m}$ is called "pseudo-Lipschitzian" at $(x, p)$, where $p \in \mathbb{R}^{d}, x \in \Gamma(p)$, if there exist neighbourhoods $U$ of $p$ and $V$ of $x$, and a constant $L \geqslant 0$ such that

$$
\left(\Gamma\left(p_{1}\right) \cap V\right) \subset \Gamma\left(p_{2}\right)+L\left\|p_{1}-p_{2}\right\| B_{m} \text { for all } p_{1}, p_{2} \in U .
$$

Proposition 5.3. Let $\mu \in \mathscr{P}\left(\mathbb{R}^{s}\right), p_{0} \in(0,1), x_{0} \in C_{p_{0}}(\mu)$ and $\mathscr{B}_{0}:=\left\{X^{-}(x): x \in \mathbb{R}^{m}\right\}$ $\subset \mathscr{B}\left(\mathbb{R}^{s}\right)$. Assume that the multifunction $\Gamma(p):=C_{p}(\mu) \quad(p \in \mathbb{R})$ is pseudoLipschitzian at $\left(x_{0}, p_{0}\right)$. Then there are a neighbourhood $V$ of $x_{0}$ and constants $L>0$ and $\delta_{0}>0$ such that for every $\nu \in \mathscr{P}\left(\mathbb{R}^{s}\right)$ with $\alpha_{\mathscr{B}_{0}}(\mu, \nu)<\delta_{0}$,

$$
\begin{aligned}
& \left(C_{p_{0}}(\mu) \cap V\right) \subset C_{p_{0}}(\nu)+L \alpha_{\mathscr{B}_{0}}(\mu, \nu) B_{m}, \\
& \left(C_{p_{0}}(\nu) \cap V\right) \subset C_{p_{0}}(\mu)+L \alpha_{\mathscr{B}_{0}}(\mu, \nu) B_{m} .
\end{aligned}
$$

Proof. Since the multifunction $\Gamma$ is pseudo-Lipschitzian at $\left(x_{0}, p_{0}\right)$, there are a neighbourhood $V$ of $x_{0}$ and positive constants $L$ and $\delta_{0}$ such that for all $p_{1}, p_{2} \in \mathbb{R}$, with $p_{1} \leqslant p_{2}$ and $\left|p_{i}-p_{0}\right|<\delta_{0}(i=1,2)$,

$$
\left(C_{p_{1}}(\mu) \cap V\right) \subset C_{p_{2}}(\mu)+L\left|p_{1}-p_{2}\right| B_{m} .
$$


(We may assume w.l.o.g. that $p_{1} \leqslant p_{2}$, since $C_{p_{1}}(\mu) \subset C_{p_{2}}(\mu)$ trivially holds if $p_{1}>p_{2}$.) Let $\nu \in \mathscr{P}\left(\mathbb{R}^{s}\right)$ be such that $\alpha_{\mathscr{S}_{0}}(\mu, \nu)<\delta_{0}$, and $x \in C_{p_{0}}(\mu) \cap V$. We put $\delta:=\alpha_{\mathscr{B}_{0}}(\mu, \nu)$, $p_{1}:=p_{0}$ and $p_{2}:=p_{0}+\delta$. By (5.4) there exists $y \in C_{p_{0}+\delta}(\mu)$ such that $\|x-y\| \leqslant L \delta$. Since

$$
t_{\mu}(y):=\mu\left(\left\{z \in \mathbb{R}^{s}: y \in X(z)\right\}\right) \geqslant p_{0}+\delta,
$$

we obtain

$$
t_{\mu}(y) \geqslant p_{0}+\left|t_{\mu}(y)-t_{\nu}(y)\right| \geqslant p_{0}+t_{\mu}(y)-t_{\nu}(y) .
$$

Thus $y \in C_{p_{0}}(\nu)$, and we have shown that there exists $y \in C_{p_{0}}(\nu)$ with

$$
\|x-y\| \leqslant L \delta=L \alpha_{\mathscr{B}_{0}}(\mu, \nu)
$$

Now, let $x \in C_{p_{0}}(\nu) \cap V$ and put $\delta:=\alpha_{\mathscr{B}_{0}}(\mu, \nu), p_{1}:=p_{0}-\delta, p_{2}:=p_{0}$. It follows that

$$
t_{\mu}(x) \geqslant t_{\nu}(x)-\left|t_{\mu}(x)-t_{\nu}(x)\right| \geqslant t_{\nu}(x)-\delta \geqslant p_{0}-\delta=p_{1} .
$$

(5.4) now implies that there exists $y \in C_{p_{0}}(\mu)$ with $\|x-y\| \leqslant L \delta$. Hence, (5.3) is shown and the proof is complete.

In the following, let $\mathscr{B}_{0}$ be a class of Borel sets in $\mathbb{R}^{s}$ having the property that $\alpha_{\mathscr{B}_{0}}(\cdot, \cdot)$ is a metric on $\mathscr{P}\left(\mathbb{R}^{s}\right)$.

We are now in the position to state the main result of this section, which is an immediate consequence of Theorem 2.5 .

Theorem 5.4. Let $f: \mathbb{R}^{m} \rightarrow \mathbb{R}$ be Lipschitzian on bounded sets, $\mu \in \mathscr{P}\left(\mathbb{R}^{s}\right), p_{0} \in(0,1)$, and let the multifunction $X$ be closed and $\left\{X^{-}(x): x \in \mathbb{R}^{m}\right\} \subseteq \mathscr{B}_{0} \subset \mathscr{B}\left(\mathbb{R}^{s}\right)$. Assume that there exists a bounded open set $V$ in $\mathbb{R}^{m}$ such that $\psi_{V}(\mu)$ is a CLM set for $f$ on $C_{p_{0}}(\mu)$. Suppose $\Gamma(p):=C_{p}(\mu)(p \in \mathbb{R})$ is pseudo-Lipschitzian at each pair $\left(x_{0}, p_{0}\right)$ with $x_{0} \in \psi_{V}(\mu)$. Then the multifunction $\psi_{V}$ (from $\left(\mathscr{P}\left(\mathbb{R}^{s}\right)\right.$, $\left.\alpha_{\mathscr{B}_{0}}\right)$ to $\mathbb{R}^{m}$ ) is upper semicontinuous at $\mu$, and there exist constants $L>0$ and $\delta>0$ such that $\psi_{V}(\nu)$ is a $C L M$ set for $f$ on $C_{p_{0}}(\nu)$ and

$$
\left|\varphi_{V}(\mu)-\varphi_{V}(\nu)\right| \leqslant L \alpha_{\mathscr{B}_{0}}(\mu, \nu)
$$

holds whenever $\alpha_{\mathscr{B}_{0}}(\mu, \nu)<\delta$.

Proof. In order to apply Theorem 2.5 , we put $(T, d):=\left(\mathscr{P}\left(\mathbb{R}^{s}\right), \alpha_{\mathscr{B}_{0}}\right)$ and $M(\nu):=$ $C_{p_{0}}(\nu)$ for each $\nu \in \mathscr{P}\left(\mathbb{R}^{s}\right)$. First we show that the multifunction $\nu \mapsto C_{p_{0}}(\nu)$ is closed at $\mu$. Let $\left(\mu_{n}\right)$ and $\left(x_{n}\right)$ be sequences such that $\mu_{n} \in \mathscr{P}\left(\mathbb{R}^{s}\right), x_{n} \in C_{p_{0}}\left(\mu_{n}\right)$, for all $n \in \mathbb{N}$, $x_{n} \rightarrow x \in \mathbb{R}^{m}$ and $\alpha_{\mathscr{B}_{0}}\left(\mu_{n}, \mu\right) \rightarrow 0$ for some $\mu \in \mathscr{P}\left(\mathbb{R}^{s}\right)$. We have to show that $x \in C_{p_{0}}(\mu)$. Since the multifunction $X$ is closed, the same holds for $x \mapsto X^{-}(x)$ and, hence, we have

$$
X^{-}(x)=\bigcap_{\delta>0} \operatorname{cl}\left(\bigcup_{y \in B(x, \delta)} X^{-}(y)\right) .
$$


This implies

$$
\mu\left(X^{-}(x)\right)=\inf _{\delta>0} \mu\left(\operatorname{cl}\left(\bigcup_{y \in B(x, \delta)} X^{-}(y)\right)\right) .
$$

Now, let $\varepsilon>0$ be arbitrary, but fixed. Then there exists $\delta_{0}>0$ such that

$$
\mu\left(X^{-}(x)\right)+\frac{1}{2} \varepsilon>\mu\left(\mathrm{cl} \underset{y \in B\left(x, \delta_{0}\right)}{\bigcup} X^{-}(y)\right) .
$$

Furthermore, there exists $n_{0} \in \mathbb{N}$ such that $\alpha_{\mathscr{B}_{0}}\left(\mu, \mu_{n}\right)<\frac{1}{2} \varepsilon$ and $\left\|x-x_{n}\right\|<\delta_{0}$ for each $n \geqslant n_{0}$. Then we have that

$$
\begin{aligned}
\mu\left(X^{-}(x)\right) \geqslant & \mu\left(X^{-}(x)\right)-\mu\left(X^{-}\left(x_{n}\right)\right)-\left|\mu\left(X^{-}\left(x_{n}\right)\right)-\mu_{n}\left(X^{-}\left(x_{n}\right)\right)\right| \\
& +\mu_{n}\left(X^{-}\left(x_{n}\right)\right) \\
\geqslant & \mu\left(X^{-}(x)\right)-\mu\left(X^{-}\left(x_{n}\right)\right)-\alpha_{\mathscr{B}_{0}}\left(\mu, \mu_{n}\right)+p_{0} \\
> & -\frac{1}{2} \varepsilon-\frac{1}{2} \varepsilon+p_{0}=p_{0}-\varepsilon .
\end{aligned}
$$

Since $\varepsilon>0$ was arbitrary, this yields $x \in C_{p_{0}}(\mu)$. Using the same arguments, it also follows that $C_{p_{0}}(\nu)$ is a closed subset of $\mathbb{R}^{m}$, for every $\nu \in \mathscr{P}\left(\mathbb{R}^{s}\right)$. Hence hypothesis (ii) of Theorem 2.5 holds. From Proposition 5.3 we obtain that the multifunction $\nu \mapsto C_{p_{0}}(\nu)$ (from $\mathscr{P}\left(\mathbb{R}^{s}\right)$ to $\mathbb{R}^{m}$ ) is pseudo-Lipschitzian* at each pair $\left(x_{0}, \mu\right) \in$ $\psi_{V}(\mu) \times\{\mu\}$ (see Definition 2.4). Furthermore, condition (iv) of Theorem 2.5 is satisfied with $p=1$. Hence, Theorem 2.5 yields the desired result.

Theorem 5.4 may also be viewed as a qualitative stability result with respect to (small) perturbations of $\mu$ in the metric space $\left(\mathscr{P}\left(\mathbb{R}^{s}\right), \alpha_{\mathscr{B}_{0}}\right)$ (and in the metrizable space $\left(\mathscr{P}\left(\mathbb{R}^{s}\right), \tau_{w}\right)$ if $\mathscr{B}_{0}$ is a $\mu$-uniformity class (cf. Remark 5.2)). Of course, the topology on $\mathscr{P}\left(\mathbb{R}^{s}\right)$ generated by $\boldsymbol{\alpha}_{\mathscr{B}_{0}}$ becomes coarse if $\mathscr{B}_{0}$ (and, hence, the set of all preimages $\left.X^{-}(x)\left(x \in \mathbb{R}^{m}\right)\right)$ is rich.

Next we show how to verify that the multifunction $\Gamma(p):=C_{p}(\mu)$ (from $\mathbb{R}$ to $\mathbb{R}^{m}$ ) is pseudo-Lipschitzian at some pair $\left(x_{0}, p_{0}\right)$, where $x_{0} \in C_{p_{0}}(\mu)$. The following result provides a corresponding criterion for the multifunction

$$
p \mapsto \Gamma(p):=\left\{x \in \mathbb{R}^{m}: g(x, p) \leqslant 0\right\},
$$

where $g$ is a real-valued function defined on $\mathbb{R}^{m} \times \mathbb{R}$. This result is, in fact, a special case of much more general results by Rockafellar [47].

Lemma 5.5. Let $g: \mathbb{R}^{m} \times \mathbb{R} \rightarrow \mathbb{R}$ be locally Lipschitzian and $\left(x_{0}, p_{0}\right)$ be such that $g\left(x_{0}, p_{0}\right) \leqslant 0$. If $g\left(x_{0}, p_{0}\right)=0$ then assume that for every pair $(x, p) \in \partial g\left(x_{0}, p_{0}\right)$ we have $x \neq 0$. Then $\Gamma$ (defined by (5.5)) is pseudo-Lipschitzian at $\left(x_{0}, p_{0}\right) .\left(\partial g\left(x_{0}, p_{0}\right)\right.$ denotes the Clarke generalized gradient of $g$ at $\left(x_{0}, p_{0}\right)$.)

Proof. Use Corollary 3.5 in [47] with $d:=1, C:=\{x \in \mathbb{R}: x \leqslant 0\}$ and $F:=g$.

In the following, we want to consider two particular models of chance constrained programs and show how to apply Theorem 5.4 to these cases. The first model (with 
random right-hand sides) is given by

$$
X(z):=\left\{x \in \mathbb{R}^{m}: h(x) \geqslant z\right\} \quad\left(z \in \mathbb{R}^{s}\right),
$$

where $h: \mathbb{R}^{m} \rightarrow \mathbb{R}^{s}$ is continuous. Clearly, the multifunction $X$ is measurable and even closed, and we have that

$$
X^{-}(x)=(-\infty, h(x)] \in \mathscr{B}_{\mathrm{R}} \quad \text { for every } x \in \mathbb{R}^{m}
$$

and

$$
C_{p}(\mu)=\left\{x \in \mathbb{R}^{m}: F_{\mu}(h(x)) \geqslant p\right\},
$$

where $F_{\mu}$ is the distribution function of $\mu \in \mathscr{P}\left(\mathbb{R}^{s}\right)$.

For (5.1) with $X$ given by (5.6) we have the following:

Corollary 5.6. Let $f$ be Lipschitzian on bounded sets, $p_{0} \in(0,1)$ and $\mu \in \mathscr{P}\left(\mathbb{R}^{s}\right)$ with locally Lipschitz continuous distribution function $F_{\mu}$. Assume that there exists a bounded open set $V$ in $\mathbb{R}^{m}$ such that $\psi_{V}(\mu)$ is a CLM set for $f$ on $C_{p_{0}}(\mu)$. Let $h: \mathbb{R}^{m} \rightarrow \mathbb{R}^{s}$ be continuously differentiable and suppose for its Fréchet derivative $h^{\prime}$ that $\operatorname{im}\left(h^{\prime}\left(x_{0}\right)\right)=\mathbb{R}^{s}$ and

$0 \notin \partial F_{\mu}\left(h\left(x_{0}\right)\right)$ hold for every $x_{0} \in \psi_{V}(\mu)$ with $F_{\mu}\left(h\left(x_{0}\right)\right)=p_{0}$. Then, $\psi_{V}$ is upper semicontinuous (u.s.c.) at $\mu$ (as a multifunction from $\left(\mathscr{P}\left(\mathbb{R}^{s}\right), \alpha_{\mathscr{B}_{\mathbb{R}}}\right)$ into $\mathbb{R}^{m}$ ) and there are constants $L>0, \delta>0$ such that $\psi_{V}(\nu)$ is a $C L M$ set for $f$ on $C_{p_{0}}(\nu)$, and

$$
\left|\varphi_{V}(\mu)-\varphi_{V}(\nu)\right| \leqslant L \alpha_{\mathscr{B}_{R}}(\mu, \nu)
$$

holds whenever $\alpha_{\mathscr{B}_{\mathrm{R}}}(\mu, \nu)<\delta$.

Proof. In order to apply Theorem 5.4 we put $\mathscr{B}_{0}:=\mathscr{B}_{\mathrm{R}}$ and have to show that the multifunction $\Gamma$ from $\mathbb{R}$ to $\mathbb{R}^{m}$ defined by

$$
\Gamma(p):=\left\{x \in \mathbb{R}^{m}: g(x, p):=p-t_{\mu}(x) \leqslant 0\right\},
$$

where $t_{\mu}(x):=F_{\mu}(h(x))\left(x \in \mathbb{R}^{m}\right)$, is pseudo-Lipschitzian at each pair $\left(x_{0}, p_{0}\right)$ with $x_{0} \in \psi_{V}(\mu)$. Let $x_{0} \in \psi_{V}(\mu) \subset \Gamma\left(p_{0}\right) . g$ is locally Lipschitzian (from $\mathbb{R}^{m} \times \mathbb{R}$ into $\mathbb{R}$ ), since $t_{\mu}$ has this property, and it holds that $\partial g\left(x_{0}, p_{0}\right)=\left\{(x, 1): x \in \mathbb{R}^{m},-x \in \partial t_{\mu}\left(x_{0}\right)\right\}$ (see the calculus rules for the Clarke generalized gradient [11, pp.38-39]). From Theorem 2.3.10 in [11] we obtain $\partial t_{\mu}\left(x_{0}\right)=h^{\prime}\left(x_{0}\right)^{\mathrm{T}} \partial F_{\mu}\left(h\left(x_{0}\right)\right)$. Now, let $g\left(x_{0}, p_{0}\right)=$ $p_{0}-F_{\mu}\left(h\left(x_{0}\right)\right)=0$. Since $0 \notin \partial F_{\mu}\left(h\left(x_{0}\right)\right)$ holds and $h^{\prime}\left(x_{0}\right)$ maps $\mathbb{R}^{m}$ onto $\mathbb{R}^{s}$, we get $0 \notin \partial t_{\mu}\left(x_{0}\right)$. Hence, the assumptions of Lemma 5.5 are fulfilled and $\Gamma$ is pseudoLipschitzian at $\left(x_{0}, p_{0}\right)$.

Remark 5.7. Let $F_{\mu}$ be continuously differentiable on $\mathbb{R}^{s}$ and $\nabla F_{\mu}$ denote its gradient. Then $0 \notin \partial F_{\mu}(z)=\left\{\nabla F_{\mu}(z)\right\}$ holds for every $z \in \mathbb{R}^{s}$ if $\mu$ has a density which is continuous and strictly positive on $\mathbb{R}^{s}$. This fact can be proved similarly as Lemma 4.9 in [49]. For the special case of linear chance constrained problems, i.e., $f(x):=$ $c^{\mathrm{T}} x, h(x):=A x\left(x \in \mathbb{R}^{m}\right)$, where $c \in \mathbb{R}^{m}$ and $A \in L\left(\mathbb{R}^{m}, \mathbb{R}^{s}\right)$, Corollary 5.6 applies under a set of conditions which are similar to those of Corollary 4.7 in [49]. Using 
a special approach it was shown there that the (globally) optimal values are Lipschitzian at $\mu$ (with respect to the Lévy-Prokhorov metric on $\mathscr{P}\left(\mathbb{R}^{s}\right)$, cf. Remark 5.10). It is well-known that $C_{p_{0}}(\mu)=\left\{x \in \mathbb{R}^{m}: \mu((-\infty, h(x)]) \geqslant p_{0}\right\}$ is convex for every $p_{0} \in[0,1]$ if the components of $h$ are concave and the probability measure $\mu \in \mathscr{P}\left(\mathbb{R}^{s}\right)$ is quasi-concave. For an extensive study of quasi-concave measures we refer to Prekopa's work (e.g. $[40,41])$.

Secondly we consider a special linear chance constrained model (with random matrix), which was first studied by Van de Panne and Popp [42]. This model is given by

$$
X(z):=\left\{x \in \mathbb{R}^{m}: z^{\mathrm{T}} x \geqslant b\right\} \quad\left(z \in \mathbb{R}^{m}\right), b \in \mathbb{R},
$$

and $\mu \in \mathscr{P}\left(\mathbb{R}^{m}\right)$ is the multivariate normal distribution $\mathrm{N}(a, \Sigma)$, where $a \in \mathbb{R}^{m}$ and $\Sigma \in L\left(\mathbb{R}^{m}\right)$ is nonsingular. Clearly, we have that the multifunction $X$ is closed and $X^{-}(x) \in \mathscr{B}_{\mathrm{C}}$, for every $x \in \mathbb{R}^{m}$. Furthermore, as shown in [42], it holds that

$$
\begin{aligned}
C_{p_{0}}(\mu) & =\left\{x \in \mathbb{R}^{m}: \mu\left(\left\{z \in \mathbb{R}^{m}: z^{\mathrm{T}} x \geqslant b\right\}\right) \geqslant p_{0}\right\} \\
& =\left\{x \in \mathbb{R}^{m}: \Phi^{-1}\left(p_{0}\right) \sigma(x)+b-a^{\mathrm{T}} x \leqslant 0\right\}
\end{aligned}
$$

where

$$
\sigma(x):=\langle\Sigma x, x\rangle^{1 / 2}=\left(\sum_{i, j=1}^{m} \sigma_{i j} x_{i} x_{j}\right)^{1 / 2} \quad\left(x \in \mathbb{R}^{m}\right)
$$

and $\Phi$ denotes the standard normal distribution function, $p_{0} \in(0,1)$. Given $c \in \mathbb{R}^{m}$ we now consider the problem

$$
\min \left\{c^{\mathrm{T}} x: x \in C_{p_{0}}(\mu)\right\} .
$$

Lemma 5.8. $C_{p_{0}}(\mu)$ is a convex subset of $\mathbb{R}^{m}$ if $p_{0} \in\left[\frac{1}{2}, 1\right)$. If $p_{0} \in\left(\frac{1}{2}, 1\right), b>0$ and (5.7) has a global minimizer, then this minimizer is unique.

Proof. The first assertion was shown in [42, pp. 421-422]. Let $p_{0} \in\left(\frac{1}{2}, 1\right), b>0$ and assume that (5.7) has two global minimizers $x, y \in C_{p_{0}}(\mu), x \neq y$. Hence, $\{\alpha x+$ $(1-\alpha) y: \alpha \in[0,1]\}$ is contained in the solution set of $(5.7)$ and it holds that

$$
\Phi^{-1}\left(p_{0}\right) \sigma(\alpha x+(1-\alpha) y)+b-a^{\mathrm{T}}(\alpha x+(1-\alpha) y)=0
$$

for each $\alpha \in[0,1]$. This implies that for each $\alpha \in[0,1]$,

$$
\sigma(\alpha x+(1-\alpha) y)=\alpha \sigma(x)+(1-\alpha) \sigma(y)
$$

holds. Since $\Sigma$ is nonsingular, $\sigma(\cdot)$ is a norm on $\mathbb{R}^{m}$. Hence, the latter equality can only be true if there is a constant $K \in \mathbb{R}$ such that $x=K y$. From $\Phi^{-1}\left(p_{0}\right) \sigma(K y)+b=$ $K a^{\mathrm{T}} y$, we obtain that $K>0$. Hence it follows that $K \Phi^{-1}\left(p_{0}\right) \sigma(y)+b=K a^{\mathrm{T}} y$ and, thus, $b=K b$. This contradicts $x \neq y$. 
Corollary 5.9. Let $b>0, p_{0} \in\left(\frac{1}{2}, 1\right)$ and assume that (5.7) has a global minimizer $x^{*} \in C_{p_{0}}(\mu)$. Let $V:=B\left(x^{*}, r\right)$ for some $r>0$. Then $\psi_{V}$ is u.s.c. at $\mu$ (from $\left(\mathscr{P}\left(\mathbb{R}^{m}\right)\right.$, $\left.\alpha_{\mathscr{B}_{C}}\right)$ to $\left.\mathbb{R}^{m}\right)$ and there exist $L>0$ and $\delta>0$ such that $\psi_{V}(\nu)$ is a CLM set for $c^{\mathrm{T}} x$ on $C_{p_{0}}(\nu)$ and

$$
\left|\varphi_{V}(\mu)-\varphi_{V}(\nu)\right| \leqslant L \alpha_{\mathscr{B}_{C}}(\mu, \nu)
$$

holds whenever $\alpha_{\mathscr{B} C}(\mu, \nu)<\delta$. (Note that $\varphi_{V}(\mu)=c^{\mathrm{T}} x^{*}, \psi_{V}(\mu)=\left\{x^{*}\right\}$.)

Proof. For the application of Theorem 5.4 we put $\mathscr{B}_{0}:=\mathscr{B}_{\mathrm{C}}, f(x):=c^{\mathrm{T}} x$ and note that $\psi_{V}(\mu)=\left\{x^{*}\right\}$ is a CLM set for (5.7). It remains to show that the multifunction

$$
p \mapsto\left\{x \in \mathbb{R}^{m}: \Phi^{-1}(p) \sigma(x)+b-a^{\mathrm{T}} x \leqslant 0\right\}=C_{p}(\mu)
$$

is pseudo-Lipschitzian at $\left(x^{*}, p_{0}\right)$. To prove this we define a function $g: \mathbb{R}^{m} \times \mathbb{R} \rightarrow \mathbb{R}$ by

$$
g(x, p):=\left\{\begin{array}{ll}
b-a^{\mathrm{T}} x, & p<0.5, \\
\Phi^{-1}(p) \sigma(x)+b-a^{\mathrm{T}} x, & p \in[0.5,1-\varepsilon], \\
\Phi^{-1}(1-\varepsilon) \sigma(x)+b-a^{\mathrm{T}} x, & p>1-\varepsilon,
\end{array} \quad\left(x \in \mathbb{R}^{m}\right),\right.
$$

where $\varepsilon>0$ is chosen such that $p_{0}<1-\varepsilon$, and we consider the multifunction $\Gamma(p):=\left\{x \in \mathbb{R}^{m}: g(x, p) \leqslant 0\right\}(p \in \mathbb{R})$. Noting that $\Gamma(p)=C_{p}(\mu)$ for every $p$ belonging to a certain neighbourhood of $p_{0}$, it suffices to show that $\Gamma$ is pseudo-Lipschitzian at $\left(x^{*}, p_{0}\right)$. To this end we use Lemma 5.5 and note first that the function $g$ is locally Lipschitzian. Since $g$ is continuously differentiable at $\left(x^{*}, p_{0}\right)$, it remains to show that $\nabla_{x} g\left(x^{*}, p_{0}\right) \neq 0$. One has that

$$
\begin{aligned}
& \nabla_{x} g\left(x^{*}, p_{0}\right)=\Phi^{-1}\left(p_{0}\right) \nabla_{x} \sigma\left(x^{*}\right)-a, \\
& \nabla_{x} \sigma\left(x^{*}\right)=\frac{1}{\sigma\left(x^{*}\right)} \Sigma x^{*},
\end{aligned}
$$

and, hence, $\nabla_{x} g\left(x^{*}, p_{0}\right)^{\mathrm{T}} x^{*}=\Phi^{-1}\left(p_{0}\right) \sigma\left(x^{*}\right)-a^{\mathrm{T}} x^{*}=-b \neq 0$. Therefore we can apply Lemma 5.5 and the proof is complete.

Note that Corollaries 5.6 and 5.9 are qualitative stability results with respect to the topology of weak convergence, since the hypotheses on $\mu$-uniformity classes (cf. Remark 5.2) are fulfilled.

Remark 5.10. Let $\mathscr{B}_{0} \subseteq \mathscr{B}\left(\mathbb{R}^{s}\right), \mu \in \mathscr{P}\left(\mathbb{R}^{s}\right)$ and assume that there exists a constant $M>0$ such that

$$
\sup _{B \in \mathscr{B}_{0}} \mu\left((\partial B)^{\varepsilon}\right) \leqslant M \varepsilon \text { for all } \varepsilon>0
$$

(where $\partial B$ denotes the topological boundary of $B$ and $(\partial B)^{\varepsilon}$ its open $\varepsilon$-enlargement). Then

$$
\rho_{\mathscr{B}_{0}}(\mu, \nu) \leqslant \alpha_{\mathscr{B}_{0}}(\mu, \nu) \leqslant(M+1) \rho_{\mathscr{B}_{0}}(\mu, \nu)
$$


holds for every $\nu \in \mathscr{P}\left(\mathbb{R}^{s}\right)$ (Lemma 4.1 in [49]), where $\rho_{\mathscr{B}_{0}}$ denotes the LévyProkhorov type metric (w.r.t. $\mathscr{B}_{0}$ ), i.e.,

$$
\rho_{\mathscr{B}_{0}}(\mu, \nu):=\inf \left\{\varepsilon>0: \mu(B) \leqslant \nu\left(B^{\varepsilon}\right)+\varepsilon, \nu(B) \leqslant \mu\left(B^{\varepsilon}\right)+\varepsilon \text {, for all } B \in \mathscr{B}_{0}\right\} .
$$

A discussion of (5.8) for $\mathscr{B}_{0}=\mathscr{B}_{\mathrm{R}}$ and $\mathscr{B}_{0}:=\mathscr{B}_{\mathrm{C}}$ can be found in [49, Remark 4.2]. From the literature it is further known that $\rho:=\rho_{\mathscr{B}\left(\mathbb{R}^{s}\right)}$ (called Prokhorov metric on $\mathscr{P}\left(\mathbb{R}^{s}\right)$ ) can be estimated in terms of the metric $\beta[16]$ and that $\beta$ is majorized by so-called Wasserstein metrics [25]. Hence the results of the present section are available for a variety of probability metrics. For Gaussian measures the results even can be related to the parameters of the distributions since explicit formulae for Wasserstein distances are available [25].

Remark 5.11. Assume that $\mu$ is estimated by empirical measures. Then it is possible to bring together the results of Section 5 with theorems on the speed of GlivenkoCantelli convergence or with Dvoretzky-Kiefer-Wolfowitz type inequalities (cf. [22]) and one obtains also for chance constrained problems convergence rates in the spirit of those developed at the end of Section 3 (Corollary 3.4).

\section{Conclusions}

The approach to distribution sensitivity of stochastic programs followed in the present paper is based on the assumption that the underlying distribution itself as an element of a suitably metrized space of probability measures is considered as a parameter. We showed how to apply recent achievements in parametric programming (cf. Klatte $[35,36]$ ) to obtain quantitative information on stability of optimal values and optimal solutions. For optimal values of stochastic programs with linear and with quadratic recourse we established Hölder continuity (with exponent $1-1 / p$ ) with respect to the metric $\beta$ on sets of probability measures with bounded $2 p$ th moment (Theorem 3.3). In two directions this extends earlier results: also quadratic recourse is considered and for linear recourse more general models than in [49] are covered. The approach also yields upper semicontinuity of optimal solutions (Theorem 3.3). In a more general frame, this property and the continuity of optimal values have been obtained by Robinson and Wets [46] and Kall [30]. The original contributions in Sections 4 and 5 concern:

- quantitative stability results (w.r.t. weak convergence) for optimal solutions of recourse problems,

- qualitative and quantitative stability results for chance constrained problems.

In Theorems 4.3, 4.4 and 4.8 we derived (in terms of the Hausdorff distance) Hölder continuity of optimal solution sets for recourse models with special structure. The exponent of Hölder continuity is $\frac{1}{2}(1-1 / p)$ on sets of probability measures with bounded $2 p$ th moment. In Example 4.6 this exponent has been shown to be best possible. 
The crucial point in Section 5 is to investigate stability with respect to the metrics $\alpha_{\mathscr{B}}$. This permits the proof of results (upper semicontinuity of optimal solutions, Lipschitz continuity of optimal values - Theorem 5.4) which in this generality do not hold with respect to weak convergence (cf. the discussion in [30]). However, under additional hypotheses discussed in Remark 5.2 conclusions on qualitative stability w.r.t. weak convergence may be drawn. For particular models with one joint chance constraint (a nonlinear model with random right-hand side, a linear model with random technology matrix [42]) we established quantitative stability under verifiable conditions (Corollaries 5.6 and 5.9).

Concerning possible applications for our general results we discussed the situation where an incompletely known original distribution is estimated by empirical ones (Corollary 3.4, Remark 5.11). In this context it is important to mention that, in probability theory, theorems on the speed of Glivenko-Cantelli convergence, Dvoretzky-Kiefer-Wolfowitz type inequalities and rates of convergence in a number of limit theorems are formulated in terms of probability metrics $[5,14,15,22]$. In Corollary 3.4 we demonstrated how to combine such a result with the material developed in the present paper. In this way we obtained an asymptotic statistical property for optimal values of recourse problems.

\section{Acknowledgement}

We are indebted to D. Klatte (PH Halle) for many valuable discussions to find a suitable parametric programming framework for distribution sensitivity analysis. We extend our gratitude to P. Kall (Universität Zürich), G. Salinetti (Università di Roma) and A. Wakolbinger (Universität Linz) for stimulating discussions. Last but not least we thank an anonymous referee for helpful suggestions to improve the final version of the paper.

\section{References}

[1] W. Alt, "Lipschitzian perturbations of infinite optimization problems," in: A.V. Fiacco, ed., Mathematical Programming with Data Perturbations II. Proceedings of the 2nd Symposium on Mathematical Programming with Data Perturbations, Washington 1980 (Dekker, New York and Basel, 1983) pp. 7-21.

[2] J.P. Aubin, "Lipschitz behaviour of solutions to convex minimization problems," Mathematics of Operations Research 9 (1984) 87-111.

[3] A. Auslender, "Stability in mathematical programming with non-differentiable data," SIAM Journal on Control and Optimization 22 (1984) 239-254.

[4] B. Bank, J. Guddat, D. Klatte, B. Kummer and K. Tammer, Non-linear Parametric Optimization (Akademie-Verlag, Berlin, 1982).

[5] R.N. Bhattacharya and R. Ranga Rao, Normal Approximation and Asymptotic Expansions (Wiley, New York, 1976).

[6] P. Billingsley, Convergence of Probability Measures (Wiley, New York, 1968).

[7] P. Billingsley and F. Topsøe, "Uniformity in weak convergence," Zeitschrift für Wahrscheinlichkeitstheorie und Verwandte Gebiete 7 (1967) 1-16. 
[8] J. Birge and R. Wets, "Designing approximation schemes for stochastic optimization problems, in particular for stochastic programs with recourse," Mathematical Programming Study 27 (1986) $54-102$.

[9] J. Birge and R. Wets, "Sublinear upper bounds for stochastic programs with recourse," Mathematical Programming 43 (1989) 131-149.

[10] S. Chevet, "Gaussian measures and large deviations," in: A. Beck and K. Jacobs, eds., Probability in Banach Spaces IV. Lecture Notes in Mathematics No. 990 (Springer, Berlin, 1983) pp. 30-46.

[11] F.H. Clarke, Optimization and Nonsmooth Analysis (Wiley, New York, 1983).

[12] B. Cornet and J.Ph. Vial, "Lipschitzian solutions of perturbed nonlinear programming problems," SIAM Journal on Control and Optimization 24 (1986) 1123-1137.

[13] A. Donchev and H.Th. Jongen, "On the regularity of the Kuhn-Tucker curve," SIAM Journal on Control and Optimization 24 (1986) 169-176.

[14] R.M. Dudley, "The speed of mean Glivenko-Cantelli convergence," Annals of Mathematical Statistics 40 (1969) 40-50.

[15] R.M. Dudley, "Speeds of metric probability convergence," Zeitschrift für Wahrscheinlichkeitstheorie und Verwandte Gebiete 22 (1972) 323-332.

[16] R.M. Dudley, Probabilities and Metrics, Lecture Notes Series No. 45 (Aarhus Universitet, Aarhus, 1976).

[17] J. Dupačová, "Stability in stochastic programming with recourse-estimated parameters," Mathematical Programming 28 (1984) 72-83.

[18] J. Dupačová, "Stability in stochastic programming-probabilistic constraints," in: V.I. Arkin, A. Shiriaev and R. Wets, eds., Proceedings of the International Conference on Stochastic Optimization, Kiev. Lecture Notes in Control and Information Sciences No. 81 (Springer, Berlin, 1986) pp. 314-325.

[19] J. Dupačová, "Stability in stochastic programming with recourse. Contaminated distributions," Mathematical Programming Study 27 (1986) 133-144.

[20] J. Dupačová, "Stochastic programming with incomplete information," IIASA-Working-Paper WP 86-08, International Institute for Applied Systems Analysis (Laxenburg, Austria, 1986).

[21] A.V. Fiacco, Introduction to Sensitivity and Stability Analysis in Nonlinear Programming (Academic Press, New York, London, 1983).

[22] P. Gaenssler and W. Stute, "Empirical Processes: A survey of results for independent and identically distributed random variables," Annals of Probability 7 (1979) 193-243.

[23] S.J. Garstka, "Distribution functions in stochastic programs with recourse: A parametric analysis," Mathematical Programming 6 (1974) 339-351.

[24] H. Gassmann and W.T. Ziemba, "A tight upper bound for the expectation of a convex function of a multivariate random variable," Mathematical Programming Study 27 (1986) 39-53.

[25] C.R. Givens and R.M. Shortt, "A class of Wasserstein metrics for probability distributions," Michigan Mathematical Journal 31 (1984) 231-240.

[26] W.W. Hager, "Lipschitz continuity for constrained processes," SIAM Journal on Control and Optimization 17 (1979) 321-338.

[27] P.J. Huber, Robust Statistics (Wiley, New York, 1981).

[28] P. Kal1, "Approximations to stochastic programs with complete fixed recourse," Numerische Mathematik 22 (1974) 333-339.

[29] P. Kall, Stochastic Linear Programming (Springer, Berlin, 1976).

[30] P. Kall, "On approximations and stability in stochastic programming," in: J. Guddat, H.Th. Jongen, B. Kummer and F. Nožička, eds., Parametric Optimization and Related Topics. Proceedings of the International Conference in Plaue, GDR, 1985 (Akademie-Verlag, Berlin, 1987), pp. 387-407.

[31] P. Kall and D. Stoyan, "Solving stochastic programming problems with recourse including error bounds," Mathematische Operationsforschung und Statistik, Series Optimization 13 (1982) 431-447.

[32] P. Kall, A. Ruszczyński and K. Frauendorfer, "Approximation techniques in stochastic programming," in: Y. Ermoliev and R. Wets, eds., Numerical Techniques for Stochastic Optimization (Springer, Berlin, 1988) pp. 33-64.

[33] V. Kan̆ková, "Stability in the stochastic programming," Kybernetika 14 (1978) 339-349.

[34] V. Kaňková, "Uncertainty in stochastic programming," in: V.I. Arkin, A. Shiriaev and R. Wets, eds., Proceedings of the International Conference on Stochastic Optimization, Kiev. Lecture Notes in Control and Information Sciences No. 81 (Springer, Berlin 1986) pp. 393-401. 
[35] D. Klatte, "On the stability of local and global optimal solutions in parametric problems of nonlinear programming. Part I and Part II," Seminarbericht No. 75 (Humboldt-Universität Berlin, Berlin, 1985).

[36] D. Klatte, "A note on quantitative stability results in nonlinear optimization," in: K. Lommatzsch, ed., Proceedings of the 19. Jahrestagung "Mathematische Optimierung" Sellin, GDR, 1987. Seminarbericht No. 90 (Humboldt-Universität Berlin, Berlin, 1987) pp. 77-86.

[37] R. Lepp, "Discrete approximation of linear two-stage stochastic programming problem," Numerical Functional Analysis and Optimization 9 (1987) 19-33.

[38] K. Marti, Approximationen stochastischer Optimierungsprobleme (Anton Hain, Königstein, 1979).

[39] P. Olsen, "Discretizations of multistage stochastic programming problems," Mathematical Programming Study 6 (1976) 111-124.

[40] A. Prékopa, "Logarithmic concave measures with applications to stochastic programming," Acta Scientiarum Mathematicarum 32 (1971) 301-316.

[41] A. Prékopa, "Logarithmic concave measures and related topics," in: M. Dempster, ed., Stochastic Programming (Academic Press, London, 1980) pp. 63-82.

[42] C. van de Panne and W. Popp, "Minimum cost cattle feed under probabilistic protein constraints," Management Science 9 (1963) 405-430.

[43] S.M. Robinson, "Stability theory for systems of inequalities, part I: Linear systems," SIAM Journal on Numerical Analysis 12 (1975) 754-769.

[44] S.M. Robinson, "Generalized equations and their solutions, part II: Applications to nonlinear programming," Mathematical Programming Study 19 (1982) 200-221.

[45] S.M. Robinson, "Local epi-continuity and local optimization," Mathematical Programming 37 (1987) 208-223.

[46] S.M. Robinson and R. Wets, "Stability in two-stage stochastic programming," SIAM Journal on Control and Optimization 25 (1987) 1409-1416.

[47] R.T. Rockafellar, "Lipschitzian properties of multifunctions," Nonlinear Analysis, Theory, Methods and Applications 9 (1985) 867-885.

[48] R.T. Rockafellar and R. Wets, "A Lagrangian finite generation technique for solving linear-quadratic problems in stochastic programming," Mathematical Programming Study 28 (1986) 63-93.

[49] W. Römisch and A. Wakolbinger, "Obtaining convergence rates for approximations in stochastic programming," in: J. Guddat, H.Th. Jongen, B. Kummer and F. Nožička, eds., Parametric Optimization and Related Topics. Proceedings of the International Conference in Plaue, GDR, 1985 (AkademieVerlag, Berlin, 1987) pp. 327-343.

[50] G. Salinetti, "Approximations for chance-constrained programming problems," Stochastics 10 (1983) 157-179.

[51] F. Solis and R. Wets, "A statistical view of stochastic programming," manuscript, University of Kentucky (Lexington, 1981).

[52] S. Vogel, "Stability results for stochastic programming problems," Optimization (formerly a series of Mathematische Operationsforschung und Statistik) 19 (1988) 269-288.

[53] J. Wang, "Distribution sensitivity analysis for stochastic programs with complete recourse," Mathematical Programming 31 (1985) 286-297.

[54] J. Wang, "Lipschitz continuity of objective functions in stochastic programs with fixed recourse and its applications," Mathematical Programming Study 27 (1986) 145-152.

[55] R. Wets, "Stochastic programs with fixed recourse: the equivalent deterministic program," SIAM Review 16 (1974) 309-339.

[56] R. Wets, "A statistical approach to the solution of stochastic programs with (convex) simple recourse," Technical Report, University of Kentucky (Lexington, 1979).

[57] R. Wets, "Stochastic programming: solution techniques and approximations schemes," in: A. Bachem, M. Grötschel and B. Korte, eds., Mathematical Programming: the State-of-the-Art 1982 (Springer, Berlin, 1983) pp. 566-603. 